\title{
Wasserfrauen auf Abwegen: Die Transformation des Rusalka-Motivs in der polnischen und russischen Popkultur
}

\author{
ELISABETH LECHNER, STEFAN SIMONEK, MARLENA TOMALA \\ Universität Wien, Spitalgasse 2, Hof 3, A-1090 Wien \\ E-mail: elli.lechner@univie.ac.at, stefan.simonek@univie.ac.at, marlena.tomala@gmail.com
}

(Received: 6 October 2016; accepted: 12 January 2017)

\begin{abstract}
Sirens became an essential element of the literary imagination in many European literatures in Romanticism and have remained popular ever since. Also, in Russian and Polish culture, the image of the dangerously alluring and transgressive female nymph called "rusalka" is omnipresent. In this paper, the authors use a comparative approach to trace the evolution of the "rusalka" motif from its creation in the Romantic period to its transformed (and often highly sexualized) use in present-day popular culture. From works written by Pushkin, Lermontov, Mickiewicz as well as Bal'mont and Gumilev (amongst others), we move on to contemporary actualizations of the motif in the music videos and lyrics of a Russian girl group ("Фабрика"), a Polish pop performer (Doda Elektroda) and a Russian folk-metal band ("Alkonost"). We argue that the centuries-old popularity of the "rusalka" motif can be ascribed to the theme's core semantics of female transgression and adaptability that lends itself especially well to the sphere of pop and its remixing and resignifying practices.
\end{abstract}

Keywords: Rusalka, Russian literature, Russian popular culture, Polish literature, Polish popular culture

Das Motiv der Wasserfrau (und in abgestuftem Maß auch jenes des Wassermannes) oder der Waldnymphe firmiert in den europäischen Literaturen spätestens seit der Romantik des 19. Jahrhunderts als elementarer Bestandteil literarischer Imagination und hat diesen hervorgehobenen Status auch noch in der Moderne zu Beginn des 20. Jahrhunderts beibehalten - zu erwähnen wäre hier etwa Lesja Ukrajinkas aus der wolhynischen Folklore schöpfendes ukrainisches Märchendrama Лісова пісня aus dem Jahre 1911, in dem die Autorin ästhetisch überzeugend den Übergang von mythologisch ausgerichteten Vorstellungswelten in das (von Autoren wie Maurice Maeterlinck oder Hugo von Hofmannsthal wesentlich mitgeprägte) lyrische Drama des Fin de Siècle vollzog. Gleichzeitig stellte die Autorin auf diese Weise letztlich auch die implizite Nähe der beiden Formen von Imagination wie auch den grundsätzlichen Charakter der Nymphe als Figur des Übergangs unter Beweis, die zwischen verschiedenen existenziellen Bereichen zu vermitteln vermag - bisweilen freilich um den Preis des eigenen Lebensglücks. In der zweiten Hälfte des vorigen Jahrhunderts avancierte die Wasserfrau dann zusätzlich noch zur privilegierten Trägerin einer spezifisch weiblichen Weltsicht, wie Ingeborg Bachmanns bekannte Erzählung Undine geht (1961) nachdrücklich belegt. 
Eine analoge Entwicklung lässt sich in enger gefasster Weise folgerichtig auch für die russische und die polnische Kultur belegen, wo die Wasserfrau als „Rusalka“ respektive „Rusałka“ präsent ist. ${ }^{1}$ Hier (wie analog auch in der ukrainischen Literatur) lässt sich im Kontext der Romantik und deren Interesse für das Volkslied gleichsam eine Nobilitierung des Rusalka-Motivs konstatieren, das über die Form der Kunstballade Eingang in den Motivbestand der Lyrik findet und von Adam Mickiewicz, Taras Ševčenko und Aleksandr Sergeevič Puškin und damit von den drei Nationaldichtern der polnischen, ukrainischen und russischen Literatur mehrfach verwendet wird. ${ }^{2}$ Über weitere textuelle Zeugnisse, wie etwa die Lyrik Michail Jur'evič Lermontovs, Nikolaj Vasil'evič Gogol's frühe, von der ukrainischen Folklore inspirierte Erzählungen oder den 1837 in Buda herausgegebenen Almanach Русалка Днъстровая als einem zentralen Moment der westukrainischen Literatur im 19. Jahrhundert verdichtete sich die literarisierte Form des Rusalka-Motivs in einer Weise, dass auch die slawische Moderne der Jahrhundertwende in ihrem Interesse für den Mythos und das Irrationale wiederholt auf das Motiv der Wasserfrau zurückgriff - Jaroslav Kvapils Libretto für Antonín Dvořáks 1901 uraufgeführte Oper Rusalka vermag dies besonders deutlich zu belegen. Im Motivbestand der Moderne ließ sich die für den Mann Verderben bringende Wasserfrau mit der Imago der Femme fatale zusammenführen und stand so weiteren Neuinterpretationen offen. In mehreren Gedichten wichtiger Vertreter des Jungen Polen und des russischen Symbolismus findet sich die Wasserfrau entweder als Motiv im Text selbst (wie etwa in Kazimierz Przerwa-Tetmajers Cień Chopina aus dem Jahr 1891) $)^{3}$ oder sogar in den Titel gerückt, wie in Konstantin Dmitrievič Bal'monts Она как русалка (1898) oder Valerij Jakovlevič Brjusovs Русалка (1907). All diese lyrischen Texte von zentralen Vertretern der Moderne in der russischen und polnischen Literatur belegen nachdrücklich, dass sich das Motiv der Wasserfrau offenbar ohne größere Schwierigkeiten in den eigenen Motivbestand transferieren und dort mit anderen zentralen Motiven verbinden ließ. Die prinzipiell auf Wandelbarkeit und Transgression hin ausgelegte Semantik des Motivs

\footnotetext{
${ }^{1}$ Bei den Wasserwesen handelt es sich um sehr alte mythische Vorstellungen, die bereits in der Antike existierten. Das Hauptmerkmal der Wasserfrau ist ihre Vermenschlichung, und sie wird dazu gebraucht, bestimmte Weiblichkeitsbilder zu spiegeln. Die bekanntesten Wasserfrauen in der griechischen Mythologie sind die Sirenen, und das Wort „Rusalka... stammt... aus dem Mittelgriechischen ('rusalia' - 'Pfingsten') oder direkt aus dem Lateinischen ('rosalia' - 'Pfingsten', ursprünglich 'Rosenfest')" (GRÜBEL 1995: 99). Die Rusalka wird in der Gestalt einer jungen Frau mit einem Fischschwanz anstelle von Beinen überliefert. Die slawische Rusalka ist ein mythisches dämonisches Wesen, eine Gestalt, der man keine eindeutige Definition zuschreiben kann. Bei den Slawen sind die Wasserfrauen ,schöne Mädchen mit durchsichtigem Körper, weißem Kleid, langem goldenem oder rötlich blondem Haar..., das das Zentrum ihres Lebens und ihrer Kraft birgt“ (VÀŇA 1992: 11). Die wissenschaftliche Auseinandersetzung mit dem Thema setzte im Übrigen bereits im 19. Jahrhundert etwa mit Franz Ritter von Miklosich ein (vgl. MiKLOSICH 1864).

${ }^{2} \mathrm{Zu}$ einem komparatistischen Vergleich des Rusalka-Motivs bei Puškin, Mickiewicz und Ševčenko vgl. Woldan 2013, weiter gefasst zur Präsenz des Motivs in den slawischen Literaturen generell vgl. FIJAŁKOWSKI 2014.

${ }^{3} \mathrm{Zu}$ den Vertonungen dieses Gedichts vgl. GMYs 2011.
} 
der Wasserfrau erleichtert also in augenfälliger Weise dessen Integration in den jeweils epochenspezifischen Bestand an künstlerischer Imagination.

Diese Dynamik manifestiert sich nach Romantik und Moderne dann nochmals im Kontext der zeitgenössischen polnischen und russischen Popkultur, wo das Motiv der Rusalka nun nicht mehr in Gedichten, sondern entweder in Songtexten oder aber in den dazugehörigen Musikvideos auftaucht und über das spezifische Interferieren von Musik, Bild und Text, das für die Ästhetik des Musikvideos insgesamt ausschlaggebend ist, erneut die eigene Wandlungsfähigkeit unter Beweis stellt. Wie die in den folgenden Abschnitten präsentierten Videos und Songs der polnischen Sängerin Doda Elektroda, der russischen Girlband „Фабрика“ und schließlich der russischen Folk-Metal-Band „Alkonost" nachdrücklich belegen, eröffnet das Motiv der Wasserfrau auch in einem zeitgenössischen popkulturellen Verweiszusammenhang jenes semantische Potential der Grenzüberschreitung sowohl in räumlicher als auch in anthropologischer Hinsicht, das dem Motiv a priori eingeschrieben scheint, von der Popkultur nun aber (besonders in sexueller Richtung) noch stärker akzentuiert wird. In dieser Beziehung verknüpft sich die Wandlungsfähigkeit des Rusalka-Motivs mit für die Popkultur insgesamt prägenden Strategien der Um- und Neucodierung von bereits vorhandenem Material, wie sie insbesondere Diedrich Diederichsen in seinem wichtigen Aufsatz Pop - deskriptiv, normativ, emphatisch 1996 hervorgehoben hat (DIEDERICHSEN 1996: 43). Drei konkrete Neucodierungen des Rusalka-Motivs im Kontext der polnischen und ukrainischen Popkultur werden ausgehend von diesen Prämissen nun genauer fokussiert und zueinander in Relation gestellt. ${ }^{4}$

\section{Doda Elektroda: Electrode}

Das Motiv der Rusalka wurde in der polnischen Literatur des 19. wie auch des 20. Jahrhunderts mehrfach aktualisiert, so etwa in Adam Mickiewiczs Świtezianka, Świteź und Rybka, in Cyprian Kamil Norwids Marzenie oder in Bolesław Leśmians Baśń. Die Wasserfrauen, die meistens als wunderschön beschrieben werden, faszinieren nicht nur durch ihr bezauberndes Äußeres, sondern auch durch ihren Gesang, der jedoch oft zum Tode führt. Die Wasserfrau ist eines der fesselndsten Urbilder. Die Rusalka ist ein zutiefst romantisches Motiv und wurde auch von der polnischen Pop- und Rock-Sängerin Dorota Rabczewska (besser bekannt

${ }^{4}$ Aufgrund der sexuellen Aufgeladenheit sowohl des Rusalka-Motivs als auch des überwiegenden Teils der weiblichen Genderrollen, die besonders die russische postsowjetische Popkultur anzubieten hat, scheint die Präsentation von Popsängerinnen als Wasserfrau in Männermagazinen nicht weiter verwunderlich: So posierte etwa Eva Bušmina - in den Jahren 2010 bis 2013 Mitglied der höchst erfolgreichen russischsprachigen Girlband „ВИА Гра“ - als Rusalka für den „Playboy“. Der Umstand, dass bei dieser Photostrecke nicht mehr, sondern im Gegenteil weit weniger an nackter Haut zu sehen ist als bei den zahlreichen Bühnenauftritten der Band, belegt die signifikante Sexualisierung von Gruppen wie eben „ВИА Гра“. Auch in der Casting-Show „Хочу в ВИА Гру“, in der die Sängerinnen der gegenwärtigen Formation ermittelt wurden, verglich einer der Juroren die Kandidatin Erika Herceg (die dann tatsächlich in die Band aufgenommen wurde) mit einer Rusalka, vgl. https://www.youtube.com/watch?v=oVlKbOp7_Cg (04:38). 
unter ihrem Bühnennamen Doda Elektroda) verwendet, wobei Doda auf eine Rusalka mit einem Fischschwanz zurückgreift. Dorota Rabczewska hat im Jahre 2000 ihre Karriere mit der Gruppe „Virgin“ gestartet und sich 2007 dann für eine Solokarriere entschieden. Im Zuge der Zusammenarbeit mit der Gruppe und als Solosängerin hat sie viele Auszeichnungen gewonnen. Doda ist eine der kontroversesten Stars des polnischen Showbusiness. ${ }^{5}$

Im Folgenden sollen Dodas Lied Electrode, das im Jahr 2011 auf dem Album 7 pokus głównych erschienen ist, sowie das dazugehörige Musikvideo näher untersucht werden. Der Songtext wurde von Paweł Ziętara geschrieben und gemeinsam mit Łukasz Mackiewicz-Krzos vertont. Obwohl der Text des Liedes auf Polnisch gehalten ist, hat der Song einen englischen Titel, eben Electrode. ${ }^{6}$ In zwei Strophen zu je fünf Zeilen und in einem Refrain von sechs Zeilen schildert die Sängerin eine jenseitige Welt in einer magischen Atmosphäre. Auf den ersten Blick hat der Songtext mit dem Rusalka-Motiv nichts zu tun, da der Begriff „Rusalka“ als solcher nicht einmal vorkommt - analog ist dies auch bei Adam Mickiewicz in dessen Ballade Świteź der Fall. Auch der Titel Electrode verweist nicht auf das Wasserwesen. Dass Dodas Song auch mit dem Motiv der Rusalka zusammenhängt, wird erst aus dem dazugehörigen Video klar, in dem das (von Doda selbst gespielte) weibliche Wesen eindeutig Züge einer stark sexualisierten Rusalka aufweist. ${ }^{7}$ Im Songtext selbst ist unter anderem von der Hölle und der Feuchtigkeit die Rede, was mindestens einen indirekten Konnex zum semantischen Potential der Rusalka eröffnet: „W głowie mej mokry zgiełk / Powódź ludzkich myśli wciaga mnie, wciąga mnie / [...] Piekło mknie, otacza sen / Karmi problemami ludzki gniew“".

Der Mensch soll sich laut Songtext von den Problemen nicht beeinflussen lassen, sondern stark bleiben. Wegen der Probleme versinkt er, aber in der Unterwasserwelt ist ihm ein leichteres Leben garantiert. Das Lied wird aus der Perspektive der Rusalka gesungen, es spricht nur die handelnde Person, also das Wasserwesen, in der ersten Person: „I cała tonę znów“. Auch in diesen Passagen ist im Motiv des Versinkens ein Konnex zur Rusalka gegeben, dagegen findet sich im Text kein Verweis auf eine unglückliche Liebe, wie es oft in literarischen Texten, die sich mit der Wasserfrau auseinandersetzen, der Fall ist. Mit dem Fehlen der Liebesintrige fehlt dem Songtext auch ein abgeschlossenes Ende im Sinne einer Lösung des Konflikts: „I cała tonę znów, to kolejny raz / Istnień błędów czas zatapia mnie / Topię się w nim, topię się / I cała tonę znów, to kolejny raz / Istnień błędów czas zatapia mnie / Topię się w nim, topię się“.

In den Zeilen des Songtexts findet man auch reflektierende Passagen. Der Refrain ist ein Hinweis auf die ungelöste Situation: Beide Frauen (die zweite Protagonistin - ein junges Mädchen - ist nur im Video präsent) bleiben für immer in der

\footnotetext{
${ }^{5}$ Vgl. https://de.wikipedia.org/wiki/Dorota_Rabczewska bzw. http://www.eska.pl/gwiazda_bio grafia/doda/378.

${ }^{6}$ Vgl. https://pl.wikipedia.org/wiki/Electrode bzw. http://www.eska.pl/news/electrode___doda_ zobacz_nowy_teledysk_dody_i_gwiazde_jako_syrene_juz_teraz_na_eska_pl_video/83111.

${ }^{7}$ https://www.youtube.com/watch? $\mathrm{v}=\mathrm{hqF} 08 \mathrm{HXvIxY}$

${ }^{8}$ Vgl. den Songtext: http://www.tekstowo.pl/piosenka,doda,electrode.html.
} 
gleichen Lage. Über die romantische Stimmung des Liedes wird die Rusalka auch zur Symbolik der Nacht in Bezug gebracht: „Pada deszcz na nocny szept“. Die Nacht gilt als Sphäre des Chaos und des Unbewussten. Sie reduziert sich auf eine lunare Sphäre des Traums, Dunkelheit und Nacht bilden eine unterirdische Welt. Die Dunkelheit erscheint als ein reduziertes Licht und als das Böse. Das Schwarze fungiert als auslöschende Kraft und verbindet die Nacht mit der Erde. Im Songtext gibt es anders als im dazugehörigen Videoclip keine Schilderung der Natur.

Doda Elektroda greift in ihrem Video, wie bereits erwähnt, das Rusalka-Motiv auf und integriert es direkt in die Handlung, was aus dem Songtext höchstens indirekt eben über die zuvor erwähnten Motive von Feuchtigkeit und Versinken hervorgeht. Das Musikvideo, in dem die Sängerin als Wasserwesen mit Fischschwanz auftritt, wurde zum ersten Mal am 7. Mai 2013 gezeigt. Die Rusalka im Musikvideo verfügt über die Macht der Elektrizität in Bezug auf den Titel des Songs. Am Anfang des Clips sitzt die Sängerin in ihrer Garderobe vor dem Spiegel und trinkt ein magisches energetisches Getränk (das keine Werbung ist), nach dem Austrinken berührt sie den Spiegel, und die schimmernden Funken aus ihrer Hand führen den Zuschauer in die märchenhafte, phantastische Welt jenseits des Spiegels hinüber, wo die Rusalka das verlorene Mädchen vor dem Tod rettet. In dem Clip wird die Rusalka im dunklen Eichenwald in einem pulsierenden Kokon geboren (01:58 bis 03:19). Das verlorene Mädchen berührt den Kokon, fällt ins Wasser und ertrinkt. In diesem Bild werden zwei Motive verknüpft - das Motiv des betrogenen Mädchens und das Rusalka-Motiv. Später fällt der Kokon ins Wasser und Doda verwandelt sich analog zu dieser Transgression zwischen den Naturelementen in eine Rusalka mit Fischschwanz, die das Mädchen rettet (03:20 bis 03:44). Interessanterweise verfügt die Rusalka über die Macht der Elektrizität, mit der das Mädchen belebt wird (bei 04:16) - vielleicht mag dies als Hinweis auf die Rolle elektrisch verstärkter Instrumente für die Popmusik insgesamt gelten. Nach der Mund-zu-Mund Beatmung wird das Mädchen mit der Elektrizität gleichsam infiziert (04:34). ${ }^{9}$ Die Szenen der Wiederbelebung des jungen Mädchens eben mithilfe der Elektrizität der Wasserfrau haben in dem Clip phantastischen Charakter. Die Elektrizität gilt als Symbol des Lebens, des Göttlichen sowie der Naturkraft und der Naturbeherrschung (BUTZER-J JACOB 2012: 93). Das Phantastische wird in die Handlung selbst verlegt. Heterogene Elemente derselben Welt - also das Natürliche und Übernatürliche, das Reale und Phantastische - sind im Video gleichberechtigt und existieren nebeneinander. Ein Blick auf die Figurenkonstellation, die ungewöhnlich ist, zeigt, dass hier die Männer (als potentielle Opfer der Rusalka) vollständig fehlen. Die Figurenkonstellation im Video ist symmetrisch, d. h. eine Figur (die von Doda gespielte Wasserfrau) steht gegen eine andere (das junge, von ihr gerettete Mädchen).

Beachtung verdient auch der Schluss des Videos: Es gibt kein tragisches Ende, sondern das Mädchen wird von der Rusalka verlassen und kehrt unter die Wasseroberfläche zurück, vollzieht also ebenfalls eine elementare Transgression in Richtung einer anderen, neuen Existenzform (04:37 bis 04:44). Das Ende des

\footnotetext{
${ }^{9}$ https://www.youtube.com/watch?v=hqF08HXvIxY
} 
Videos korrespondiert augenfällig mit den traditionellen Beschreibungen der Rusalka: „Nach dem Ende des Sommers zogen sie sich [die Wasserfrauen] in Federnester auf dem Grunde ihrer Flüsse zurück, wo sie bis zum nächsten Frühjahr überwinterten“ (Monaghan 1997: 239). Es existiert die Vorstellung, wonach die Wasserfrauen in stillen Gewässern, in der Nähe von Brunnen, im Wald oder auch in mit niedrigen Bäumen bestandenen, waldigen und zumeist sumpfigen Gegenden leben, was auch in dem Video ersichtlich ist.

Ziel des Videos war es, das Publikum zu schockieren und zu überraschen. Die Sängerin hat dafür auch selbst einige Unterwasserszenen aufgenommen. Dieser Clip, der neun Tage lang gedreht wurde, beinhaltet viele romantische Elemente, wie das Wasser, die Nacht am Ufer, den Mondschein oder die Unterwasserwelt mit der Rusalka und ihrem Gesang. Es sind viele symbolische Elemente zu finden, z. B. der Kokon, der eine Schutzfunktion hat und eine Transformation sowie eine Entwicklung symbolisiert. Das Wasser spielt eine wichtige Rolle, weil es eine schöpferische Kraft ist. Das Wasser ist Symbol der Erneuerung und stellt die Quelle der Nixe und ihrer Unsterblichkeit dar. Durch das Wasser kann die Rusalka zur Sonne und zu den Sternen hinauf blicken. Im Video findet man eine deutliche Bewegung nach oben und nach unten als Gegensatz zwischen Himmel und Erde. Im Wasser wird die Verbindung zwischen Leben und Tod herauskristallisiert. Die Rusalka fungiert als Vermittlerin zwischen Himmel und Erde. Ambivalenz tritt hier in der Gegenüberstellung von Wasser und Festland respektive von Oben und Unten zutage. Es ist eine Welt, die eine Wechselwirkung zwischen einem Menschen - dem jungen Mädchen - und der Rusalka manifestiert, die über ihre jeweilige Zugehörigkeit zu verschiedenen Elementen (Erde und Wasser) auch unterschiedliche Dimensionen der Wirklichkeit verkörpern. Weiter ist dies auch eine Welt, die durch eine romantische Stimmung mit dem Rusalka-Motiv generell in Verbindung steht und von diesem beherrscht wird.

Das Video verweist auf die Nähe zur Natur, die beide Protagonistinnen umfängt. Mit einer Ortsbestimmung (dem dunklen Wald) wird auch die dämonische Welt der Wassergeister eingespielt. Der Wald ist ein verzauberter Ort des Geheimnisses, der Verirrung und unheimlicher Begegnungen; er bildet den Übergang zur Wasser- und Unterwelt und gilt als Symbol der Verborgenheit und Täuschung sowie des Ursprünglichen und der Freiheit (BUtZER-JACOB 2012: 470). Das junge Mädchen aus dem Wald und die Gestaltung der Handlung im Video verweisen auf eine mögliche Ausformung der Rusalka als Waldnixe und auf die Elemente der Nacht, des Mondes, des Wassers und der Eichenblätter. Der Widerstandsgeist und die Stärke, durch die das Auftreten der Rusalka markiert ist (beides symbolisiert durch die Eiche, vgl. Butzer-JACOB 2012: 84), unterstreichen dies. Dieses Phänomen ist analog auch am Beginn von Mickiewiczs Ballade Świtezianka präsent: „Brzegami sinej Świtezi wody / Idą przy świetle księżyca“ (MıcKIEwICz 1953: 19). Im Clip fungiert der Mondschein als die Sonne der Wassergeister, und der Glanz des Mondes hat eine verzaubernde Wirkung auf die Protagonistinnen des Videos.

Das solare Prinzip ist durch die Präsenz der Farbe Gold im Video stark ausgeprägt. Eine wichtige Rolle spielt hier auch die Assoziation von Wasser und Nacht, 
Tiefe und Finsternis mit der weiblichen Sphäre. Auffällig scheint auch die reflektierende Funktion des Wassers als Spiegel für die (weibliche) Luna. Nicht der Tag, der die Menschen belebt und die kranken Seelen heilt, sondern die Nacht erweist sich als die Wirklichkeit. Die Innenwelt steht im Einklang mit der nächtlichen Natur, sie garantiert den Protagonistinnen die Teilhabe an der Wahrheit. Die Nacht befreit die schöpferischen Kräfte der Phantasie, sie eröffnet den Zugang zum Unbewussten. In der Natur finden die Gestalten Schönheit und geistige Nahrung. Bei näherer Betrachtung fällt jedoch auf, dass es sich im Video um keine reine und objektive Naturschilderung handelt, sondern dass es primär um Bewegung und das Überschreiten von Grenzen geht. Mit dem Songtext und dem dazugehörigen Video hat die Popsängerin die Aufmerksamkeit des Publikums geweckt. Die polnische Sängerin Doda benützt das Rusalka-Motiv zum einen, um eine romantische Stimmung zu gestalten, und zum anderen, um das Publikum mit ihrem Song und dem dazugehörigen Video zu faszinieren, was ihr auch durchaus gelungen ist. ${ }^{10}$

Es ist besonders überraschend, dass die textuelle Ebene nicht vollständig auf die visuelle hin abgestimmt ist, da die Wasserfrau erst am Ende des Videos tatsächlich im Wasser untertaucht. Im Songtext wird dies mehrmals erwähnt: „I cała tonę znów, to kolejny raz". Im Video ist schon die Ausgangssituation anders als im Songtext: Im Clip sieht man die Hauptprotagonistin, die den Spiegel berührt und durch die schimmernden Funken in die phantastische Welt hinübergetragen wird, im Songtext dagegen ist in der ersten Sequenz vom Regen und den menschlichen Gedanken die Rede. Man findet auch einen Unterschied in der Figurenkonstellation, denn ungeachtet dessen, dass im Lied von dem jungen Mädchen keine Rede ist, taucht es im Videoclip sehr wohl in zentraler Rolle auf. Die Sängerin präsentiert eine romantisch-unheimliche Welt, die sich vor den Augen des Zuhörers und des Zuschauers entfaltet, und erst aus der Handlung des Videoclips heraus wird klar, wer die beiden Figuren eigentlich sind. Im Video wird deutlicher als im Songtext die konkrete Lokalisierung der Cliphandlung am Ufer eines Flusses dargestellt.

Interessant ist, wie Dodas Songtext und auch das zugehörige Musikvideo mit der Tradition der Rusalka aus der polnischen Literatur korrespondieren. In Mickiewiczs Ballade Świteź etwa hat das Wasserwesen eindeutig Züge einer Rusalka: „nie straszydło wcale, / Żywa kobieta w niewodzie, / Twarz miała jasną, usta jak korale, / Włos biały skąany w wodzie“ (Mickiewicz 1953: 14). ${ }^{11}$ In der Świtezianka ist sie folgendermaßen dargestellt: ,Jej twarz jak róży bladej zawoje, / Skropione jutrzenki łezką; / Jako mgła lekka, tak lekkie stroje / Obwiały postać niebieską" (MicKIEwICz 1953: 21). Hier findet man nun eine konventionelle Rusalka,

${ }^{10}$ Auch auf der Konzertbühne griff die Sängerin offenbar auf die Rolle der Rusalka zurück und trat dementsprechend live in einem Kostüm auf, das in einem Fischschwanz endet, vgl. https://www. youtube.com/watch? $v=$ wKiEm_jqLfE.

${ }^{11}$ In der Ballade erhält die Wasserfrau insofern eine neue Funktion zugesprochen, als sie über die Belagerung der litauischen Stadt Świteź durch die Truppen des russischen Zaren berichtet (vgl. WOLDAN 2013: 444), dafür fehlen hier die erotischen Motive (vgl. ZGORZELSKI 1976: 177). Die romantische Bildhaftigkeit manifestiert sich in den Blumen und den Wasserfrauen, die den Świteź-See beschwören (vgl. FABRE 1956: 56). 
die vom Dichter umgestaltet wird: Sie ist ein reines Fabelwesen aus dem Wasser, das keine irdische Präexistenz hat und sich in verschiedenen Inkarnationen zeigen kann, weil sie einmal Mädchen aus dem Wald und dann wieder Nixe aus dem Wasser ist (vgl. Woldan 2013: 448). In der Ballade Rybka heißt es: „Wtem rybią łuskę odwinie, / Spojrzy dziewicy oczyma; / Z głowy jasny włos wypłynie, / Szyjka cieniuchna się wzdyma. // Na licach różana krasa, / Piersi jak jabłuszka mleczne, / Rybią ma płetwę do pasa“" (Mickiewicz 1953: 27). Bei Norwid wird das Wasserwesen als ,czarowna dziewica“ bezeichnet und mit dem Traum gleichgesetzt: „Jam Rusałka-marzenie - to młode marzenie“ (NorwID 2000: 25).

In Dodas Clip findet man (korrespondierend mit dem Frauenbild der Popkultur) eine extrem sexualisierte Gestalt des Wasserwesens, die Schönheit und Vitalität reflektiert. Das Medium des Videos wird hier eindeutig dazu benutzt, um das traditionelle Motiv der Rusalka neu und anders zu gestalten. Neu im Kontext des Rusalka-Motivs ist auch die Funktion, die die Frau aus dem Wasser ausübt: Sie belebt das Mädchen mit der Kraft der Elektrizität. Diese Szene ruft den Eindruck hervor, als ob das Rusalka-Motiv hier in einen zeitgenössischen technischen Kontext eingebettet werden soll. Es geht im Video nicht um die Rache der betrogenen Frau, sondern um die Wiederbelebung des Mädchens. Das Rusalka-Motiv scheint hier in eine technisierte Gegenwart transferiert, und das Wasserwesen beansprucht symbolische wie popkulturelle Bedeutung gleichermaßen.

In den Werken der polnischen Dichter wird die Rusalka als Rächerin dargestellt, wie etwa in der Świtezianka von Mickiewicz: ,A dusza przy tym świadomym drzewie / Niech lat doczeka tysiąca, / Wiecznie piekielne cierpiąc żarzewie / Nie ma czym zgasić gorąca“" (Mickiewicz 1953: 24). ${ }^{12}$ Auch in der Ballade Świteź ist von Rache die Rede: „Młodzieńcy, wiecie, że tutaj bezkarnie / Dotąd nikt statku nie spuści, / Każdego śmiałka jezioro zagarnie / Do nieprzebrnionych czeluści“ (14). ${ }^{13}$ In Rybka wiederum wird die Rache der Rusalka durch die Verwandlung des Mannes und seiner Frau in Stein sichtbar: ,Sterczał wielki głazu kawał / I dziwną kształtu budową / Dwa ludzkie ciała udawał" (29). In allen Gedichten findet man als tragisches Ende den Tod des ungetreuen Liebhabers, an dem sich die Rusalka rächt. ${ }^{14}$

${ }^{12}$ Es handelt sich um eine spezifische Strafe, und in dieser Kontamination von Wasser und Feuerqualen wird der freie Umgang des Autors mit Versatzstücken aus der Folklore gezeigt (vgl. WOLDAN 2013: 449).

${ }^{13}$ Als Instrument der Strafe gelten hier die in Blumen verwandelten Frauen und Mädchen. Nachdem die Truppen die Blumen gepflückt hatten, starben sie an deren Gift. „Das Motiv der Verwandlung, das im Zentrum der Botschaft der Rusalka aus dem Świteź-See steht, verweist nicht nur auf den mythologischen Kern historischer Traditionen und den für die Folklore typischen Glauben an das Wunderbare, sie steht auch im Zentrum des Rusalka-Texts als solchem: ein irdischer Mensch wird in ein dämonisches Wasserwesen verwandelt, oder auch umgekehrt..." (WOLDAN 2013: 446).

14 „Mickiewicz benutzt das Rusalka-Motiv zum einen, um lokale historisch-legendäre Traditionen zu gestalten, zum anderen auch um seine programmatischen Ansichten zur romantischen Dichtung und Weltanschauung zu äußern“ (WolDAN 2013: 452). Laut Alina Witkowska und Ryszard Przybylski beinhalten die Balladen von Mickiewicz die moralische Problematik von Schuld und Strafe sowie von Gewissen und Verantwortung für das eigene Handeln. Gleichzeitig finde in den Balladen eine Annäherung zwischen Ethik und Phantastik statt (WITKOWSKA-PrZYBYLSKI 1997: 250). 
Die Rusalka im Video wird nicht zum üblichen Typus der Rächerin gemacht, sondern zu einem anderen Typus, nämlich dem der Rettungsschwimmerin (und damit zu einer weitläufigen Verwandten von Pamela Anderson in der Serie „Baywatch"). Die Wasserfrau aus dem Clip verfügt weiter über eine gänzlich andere Macht als das unter anderem eben von Mickiewicz literarisierte Wasserwesen, nämlich über die der Elektrizität. Bei Mickiewicz dagegen manifestiert sich die Macht der Rusalka auf traditionelle Weise in Form des Kitzelns: „Z lekka mu w stopy załechce. // I tak go łechce, i tak go znęca“ (Mickiewicz 1953: 22), und die Wasserfrau selbst hat verführerische Kraft und ruft: „I do mnie, woła, pójdź do mnie“" (MickIEWICZ 1953: 22). Bei Norwid wiederum vermag sie zu bezaubern: „Zostań - a ja tymczasem polecę daleko / I zacznę moje czary - bo też umiem cuda, / Jakie stwarzać nie każdej rusałce się uda“ (NorwiD 2000: 26). Bei Bolesław Leśmian gilt das Lachen als wirkende Kraft der Rusalka: „Lecz piersi im rozpiera / Śmiech pusty i uparty“ (LEŚMIAN 2015: 594). In denjenigen Gedichten, in denen eine Wasserfrau auftaucht, wird auch die landschaftliche Szenerie genauer beschrieben: „Strzeliło srebrne słońce / Nad siną gór koronę, / Obłokiem purpurowym / Na dwoje przekreślone. / U samych progów lasu / Lśnią rzeki blade tonie“ (LEŚMIAN 2015: 594).

Wenn nun derartige Naturschilderungen in Doda Elektrodas Songtext fehlen, so mag auch dies als Indiz für die kommerzialisierte Form der Neuausrichtung der Rusalka dienen - Doda übernimmt zwar die tradierte Figur der Wasserfrau, legt die Betonung aber weniger auf die Weiterführung der literarischen Tradition von der Romantik bis hin zum Jungen Polen (obwohl die medialisierten Formen von Popkultur auch eine derartige Bezugnahme durchaus ermöglichen würden), sondern kehrt entsprechend ihrem eigenen Image eindeutig das sexuelle Potential der Rusalka-Figur hervor.

\section{„Фабрика“": Я тебя зацелую}

Ähnlich wie in der polnischen Popkultur finden sich auch in der russischsprachigen Popwelt des 21. Jahrhunderts Aktualisierungen des Rusalka-Motivs. Inspiriert sind diese Neuinterpretationen der Wasserfrauen-Thematik in Zeiten der Globalisierung aber schon lange nicht mehr nur von den kanonisch tradierten mythologischen Rusalki aus Bildender Kunst und Literatur, wie sie etwa von Michail Aleksandrovič Vrubel' bzw. von Aleksandr Sergeevič Puškin und Michail Jur'evič Lermontov gestaltet wurden, sondern sicherlich auch von global verbreiteten und konsumierten Märchen, wie Hans Christian Andersens Die kleine Meerjungfrau (1837) und deren Adaptionen (etwa Walt Disneys The Little Mermaid, 1989). Im russischsprachigen Kulturraum sind die Wasserfrauen in vielfältiger Weise präsent - man findet die verführerischen Nixen als Werbetestimonials für Kleidung, Kosmetika und vieles mehr allgegenwärtig in den Medien, als Protagonistinnen in phantastisch motivierten Filmen (vgl. den Spielfilm Русалка der Regisseurin Anna Melikjan aus dem Jahr 2007) und in Musikvideos erfolgreicher Popgruppen, wobei sich die folgenden Ausführungen besonders mit dem 2010 veröffentlichten 
Videoclip Я тебя зацелую der gecasteten Girlband „Фабрика“ und mit dessen Einbettung in den Rusalka-Diskurs beschäftigen. ${ }^{15}$

Vorerst soll also eine literarisch-kulturelle Kontextualisierung vorgenommen werden, die belegt, dass (pop-)kulturelle oder literarische Aktualisierungen von Wasserfrauen-Mythen in Russland keine Neuerfindung des 21. Jahrhunderts darstellen. Vielmehr handelt es sich bei diesen „Updates“ oder - um in der Terminologie der Popkultur zu bleiben - bei diesen „Remixes“16 des Motivs um ein konsequentes Fortschreiben verschiedenster Diskurse rund um Weiblichkeit, Sexualität, Verführung, Macht und Gewalt, die schon lange im russischsprachigen Kulturraum präsent sind - besonders aber seit der Blütezeit der Romantik, in der man sich auf die eigene nationale Volksmythologie besann und sie statt antiker Vorbilder in seinen Werken verarbeitete. Maria Deppermann verweist ebenfalls auf diese Kontinuität im Rusalka-Diskurs, indem sie konstatiert, dass Puškin, Lermontov und Gogol' mit der Eingliederung der „Rusalka als Archetyp der nicht kanonisierten Frau... in die Literatur... eine mythopoetische Konstante von der Romantik bis in die Moderne“ (DEPPERMANN 2006: 157) geschaffen haben.

Diese nicht kanonisierte rusalkahafte Form von sinnlicher Weiblichkeit stellt das ultimative Gegenbild zu jener Konzeption von Femininität dar, die man gemeinhin mit Russland als weiblich definiertem kulturellem Bezugsraum assoziiert. Es ist weithin bekannt, dass Russland (russisch: Россия, auch grammatikalisch weiblich) oftmals als feminin bzw. als Frau bezeichnet und dargestellt wird. ${ }^{17}$ International am bekanntesten ist wohl die vermeintlich auf ,uralte[n] matriarchalische[n] Traditionen“ (NohesL 2013: 10) beruhende Zuschreibung „Mütterchen Russland", die laut Regine Nohejl im Laufe des 19. Jahrhunderts als Reaktion auf die Exotisierung und Orientalisierung (d. h. immer auch Verweiblichung) Russlands durch den Westen entstand:

Im Laufe des 19. Jahrhunderts entwickeln russische Intellektuelle aber auch wesentlich raffiniertere, komplexere Strategien im Umgang mit den westlichen Fremdbildern. Man greift diese auf, allerdings nicht im Sinne einer bloßen masochistischen Identifizierung mit dem Status des Weiblich-Unterlegenen oder Barbarisch-Wilden, sondern im Sinne einer affirmativen Um- und Aufwertung solcher Stereotypen. Das Weiblich-Ursprüngliche wird wie in einem Vexierbild umdefiniert vom Makel Russlands zum Defizit des Westens (NOHEJL 2013: 10).

Was im Russland des 21. Jahrhunderts von diesem Weiblichkeits- und Mütterlichkeitskult übrig bleibt, ist nicht zuletzt der kommerziell motivierte Ausverkauf industriell gefertigter Matrjoschka-Puppen, die, obwohl sie mit alten Mythen und

${ }^{15} \mathrm{http}: / /$ www.youtube.com/watch?v=QCWdo8NBg7g

${ }^{16}$ Vgl. zur Relevanz der Remix-Technik den amerikanischen Medientheoretiker Lev Manovich: „Die ganze Praxis der elektronischen Popmusik in den letzten drei Jahrzehnten ist ein Beweis dafür, welche Macht neue Technologien haben, neue komplexe und reiche Remixe zwischen unterschiedlichen Kulturen, Stilen und Empfindungsvermögen zusammenzuschweißen“ (MANOVICH 2005: 80).

${ }^{17}$ Zum Konnex von Russland und Weiblichkeit vgl. HubBs 1988 sowie RutTEN 2010, zu Russland und Genderdiskursen allgemein vgl. NOHEJL 2013. 
Kunsthandwerk nur mehr wenig gemein haben, metonymisch für das Land, die Kultur und für die Traditionen Russlands stehen. Sind die Figuren auch Ausdruck einer von Konsum geprägten postsowjetischen Gesellschaft, so haftet den simulakrahaften Puppen trotzdem noch immer die Konnotation von Fruchtbarkeit, Weiblichkeit und Mutterschaft an (vgl. dazu auch HubBs 1988: XI-XVII). Eine solche idealtypische (und gleichzeitig sehr restriktive) Form von Weiblichkeit - die fürsorgliche, liebende Mutter - die in der Matrjoschka verkörpert wird, steht also repräsentativ für Russland als Heimat und Zuhause. Diese konservative Konzeption von Femininität, die sich über Mutterschaft und Häuslichkeit definiert, wird von der patriarchalen Gesellschaft Russlands bis heute offensiv unterstützt. Während in Zeiten der Sowjetunion der Versuch der Androgynisierung, „der Herstellung des ,neuen', universalen Menschen“ (NOHEJl 2013: 12), unternommen und die Vermännlichung der Frau angestrebt wurde, verlieren diese Ansprüche nach dem Zerfall des kommunistischen Systems in den 1990er Jahren wieder an Wichtigkeit; nach einer kurzen Periode der (zivil-)gesellschaftlichen Öffnung (vgl. GoDEL 2002, GaRSTENAUER 2010) kommt es zu einem ,geradezu kitschige[n] Revival des Zweigeschlechtermodells... - der hegemoniale Macho und die schöne Puppe" (NoHEJL 2013: 14). Wie sich bei der Analyse von $Я$ тебя зацелую von „Фабрика“ јеdoch noch zeigen wird, steckt hinter der Fassade der ,schönen Puppe“ oft mehr Widerständiges und Rusalkahaftes, als es auf den ersten Blick den Anschein hat.

Im Russland Vladimir Putins, der sich als hypermaskuline Führungspersönlichkeit inszeniert, ist also, zusammenfassend, eine Rückkehr zu überkommenen, sehr eingeschränkten Formen von Weiblichkeit und weiblicher Sexualität unübersehbar: ${ }^{18}$ In Politik und Wirtschaft spielen Frauen eine untergeordnete Rolle, oftmals können sie nur im Rahmen der (Pop-)Kultur ihre Identitäten im öffentlichen Raum verorten (vgl. LECHNER 2014: 14-16 bzw. 42-45). Dementsprechend kommt nicht nur der Popkultur eine wichtige, identitätsstiftende Rolle für Frauen in Russland zu, die abseits davon kaum kritische Ausdrucksmöglichkeiten in der Öffentlichkeit haben (vgl. die harschen Strafen für den feministischen Protest von „Pussy Riot" im Jahr 2012), sondern auch dem aktualisierten Motiv der Rusalka, da die Figur der attraktiven, verführerischen Wassernixe in krassem Gegensatz zur häuslichen Femininität der die Nation verkörpernden Matrjoschka steht, die vom Staat als einem klar maskulin definierten Bereich propagiert wird.

Jost Eickmeyer und Sebastian Soppa versuchen im Vorwort des von ihnen herausgegebenen Sammelbands Umarmung und Wellenspiel das Phänomen Wasserfrau interkulturell zu fassen und zu definieren (EICKMEYER-SOPPa 2006). Dabei weisen sie den sagenumwobenen Wesen ein Grenzgänger-Dasein,,im Schattenreich von Natur und menschlicher Kultur" (EICKMEYER-SOPPA 2006: 7) zu. Besonders interessant erscheint aber der Verweis auf die gänzlich verschiedenen Charakterausprägungen der Wasserfrau: „Einmal ist sie in Gestalt der Sirenen Verderberin, ein andermal in Gestalt der kleinen Seejungfrau Lebensretterin“" (EICKMEYER-SOPPA

${ }^{18}$ Zum Personenkult um den betont maskulinen Staatschef vgl. MARTINEZ 2012, GosCiLO 2013, Marsh 2013: 204, Ritter 2006: 118, MenZel 2013, Rutten 2013, Kreß 2010: 132. 
2006: 8). Wie sich besonders anhand der Analyse literarischer Varianten des Motivs noch zeigen wird, können die zu besprechenden Rusalka-Repräsentationen jedoch keinesfalls einfach einem der diametral entgegengesetzten Endpunkte dieses Spannungsfelds zwischen Mörderin und Beschützerin zugeordnet werden. Vielmehr ist man in den kanonischen Texten konfrontiert mit emotional tiefgründigen Frauenfiguren mit persönlicher Leidensgeschichte, die von der sie umgebenden patriarchalischen Gesellschaft ausgenützt bzw. schlussendlich verstoßen und erst deshalb zu Verbrecherinnen wurden.

Der auf Rache sinnenden Rusalka Puškins und den satirisch neu gefassten Rusalki der Popband „Фабрика“ ist jedoch eines gemein - ihre sie ermächtigende Attraktivität und Sexualität. Die Rusalka verkörpert in der heutigen, wie in früheren literarischen Gesellschaften eine subversive Form von Femininität und selbstbestimmter, weiblicher Sexualität, die als unkontrollierbar, ausufernd und beängstigend wahrgenommen wird, gerade weil sie außerhalb von gesellschaftlich akzeptierten Institutionen wie der Ehe abläuft. Die Rusalka, „das ausgeschlossene Andere der Zivilisation" (EICKMEYER-SOPPA 2006: 13), muss damit immer in Konflikt stehen ,zu einer Lebensordnung, die unterdrückt, wofür die Wasserfrau steht: sinnliche Erfüllung und elementare Ungebundenheit" (EICKMEYER-SOPPA 2006: 14). Die Rusalka wird damit nicht nur zum Antonym der brav lächelnden, hausmütterlichen Matrjoschka, die als sinnbildliche Über-Mutter für die Nation Russlands steht, sondern auch zum Gegenbild der „braven russischen Braut“ (DEPPERMANN 2006: 155), der sie mit ,ihre[r] Sinnlichkeit und ungehemmte[n] Sexualität“ (DEPPERMANN 2006: 155) sowie auch der von ihr ausgehenden Gefahr entgegensteht. Die subversiven Elemente des ländlichen Rusalka-Kults werden auch von Joanna Hubbs hervorgestrichen:

The cult of the rusalki expressed women's desire for freedom and control. As spirits of unbaptized children or drowned girls who were freed by death from the constraints of patriarchal marriage, the rusalki remained forever young and at liberty, challengers to the social and religious order (HuBBs 1988: 35).

Wenn also „Фабрика“ in ihrem Video Я тебя защелую auf die mythologischen Rusalki referieren, so inszenieren sich die Sängerinnen - bewusst oder nicht als sexy Verführerinnen mit langer kulturgeschichtlicher Tradition. Wie sich bei der Beschäftigung mit dem Rusalka-Mythos in der russischen Kulturgeschichte zeigt, ist die oft zitierte und vielfach abgelehnte Sexualisierung der zeitgenössischen (Pop-)Kultur, die Vielen Grund für anhaltenden Kulturpessimismus gibt, also keineswegs ein neues Phänomen. Rusalki - sei es in der Volksmythologie, in den Werken der Romantiker oder Bildender Künstler oder eben in der zeitgenössischen Popkultur - zeichneten sich schon immer durch ihre verführerische und gefährliche Attraktivität bzw. ihren ,ästhetischen Wert“ (GRÜBEL 1995: 102) aus und tun dies noch heute.

Sieht man sich die Ästhetik des angesprochenen Musikvideos an, so fühlt man sich stark an die in der Romantik generierten Versionen der Rusalka und der sie umgebenden Natur erinnert. Besonders Puškins und Lermontovs Rusalka-Varia- 
tionen haben die Konzeption dieser mystischen weiblichen Wesen und ihrer Aufenthaltsorte entscheidend mitgeprägt. In Puškins Werk taucht die Rusalka vier Mal auf (vgl. Deppermann 2006: 157), besonders prägend für das kulturelle Verständnis der slawischen Wasserfrau sind jedoch das Gedicht Русалка (1819) und das bewegende Dramenfragment Русалка (1826-1831). Aus dem lyrischen Werk, in dem ein alter Mönch von einer sinnlichen Wassernixe verführt und um Glauben, Verstand und Leben gebracht wird, geht eindrucksvoll die Stimmung und natürliche, ländliche Umgebung hervor, die man heutzutage automatisch mit den Rusalki assoziiert: Es ist Nacht, man befindet sich am Ufer eines Sees, der Mond scheint und der Wind streicht durch die Blätter, als sich aus den Wogen eine wunderschöne, nackte Frau mit langem, nassem Haar erhebt, das sie versonnen kämmt. Diese geheimnisvolle Schöne ist von einer unbändigen Attraktivität und selbstbewusst dargebrachten, spielerischen Sexualität geprägt, die nichts von christlicher Moral wissen will: „Глядит, кивает головою, / Целует из дали шутя, / Играет, плещется волною, / Хохочет, плачет, как дитя, / Зовет Монаха, нежно стонет... / ,Монах, Монах! Ко мне, ко мне!'...“ (PUŠKIN 1974: 85). Damit vermag sie sogar einen vermeintlich zölibatär lebenden, alten Mönch zu verführen, von dem am Ende des Gedichts nur mehr der von Puškin keck eingesetzte symbolische graue Bart im See zu erkennen ist: „Монаха не нашли нигде, / И только бороду седую / Мальчишки видели в воде“ (РUŠкIN 1974: 85).

Besonders aus der Handlung des Dramas geht der entscheidende Unterschied zwischen westlichen Vorstellungen von Meerjungfrauen und den slawischen $\mathrm{Ru}-$ salki hervor. Eine Rusalka ,ist kein seelenloses Elementarwesen, das dem Wasser entsteigt, um durch die Verbindung mit einem Mann eine Seele zu erlangen“ [Hervorhebung im Original] (DEPPERMANN 2006: 153), vielmehr ist sie ,selber eine verlorene menschliche Seele“, „ursprünglich ein Mensch, eine liebende, aber betrogene, meist schwangere junge Frau, die sich aus Verzweiflung an einem Baum erhängte oder ins Wasser stürzte, dort aber als Ertrunkene keine Ruhe fand“" (DEPPERMANN 2006: 153). Mit dieser Definition Deppermanns ist auch der Inhalt von Puškins sozialkritischem Dramolett konzise zusammengefasst - ein junger Fürst hat eine Affäre mit einer mittellosen Müllerstochter, deren Herz er bricht, weil er aus Standesgründen und generellem Desinteresse eine andere heiratet. Auch der Versuch monetärer Abgeltung seines Verrats kann das Schicksal der jungen Geliebten nicht mehr ändern - die unehelich schwanger Gewordene begeht Selbstmord und sinnt als Rusalka auf Rache. Der reuegeplagte Fürst ist in seiner Ehe jedoch nicht glücklich und kehrt zurück zu den auch für ihn verhängnisvollen Ufern des Dnjepr, wo er den vor Kummer verrückt gewordenen Müller und Vater der Selbstmörderin trifft, den er aus Mitleid zu sich ins Schloss nehmen möchte. Zur selben Zeit planen jedoch die Rusalki am Grund des Dnjepr bereits Vergeltung für das Unheil ihrer Anführerin, der ehemaligen Müllerstochter, die ihre siebenjährige (!) Tochter auf ihren früheren Liebhaber ansetzen will. Vor der Umsetzung dieses inzestuösen Vorhabens bricht das Fragment Puškins jedoch ab. Aus dem gegen Ende des Dramas gehaltenen Monolog der ranghöchsten Rusalka gehen deren persönliches Leid und zutiefst zerrüttete Psyche hervor. Aus einem 
naiven, lebensfrohen Mädchen vom Land, dem in der beschriebenen patriarchalen und von Klassenunterschieden geprägten Gesellschaft kaum Rechte zustehen, ja mehrfach Unrecht durch den geldgierigen Vater und den rücksichtslosen jungen Fürsten geschieht, wird durch ihre Transformation im Tod eine kalte, aber mächtige Rusalka: „С той поры, / Как бросилась без памяти я в воду / Отчаянной и презренной девчонкой / И в глубине Днепра-реки очнулась / Русалкою холодной и могучей, / Прошло семь долгих лет - я каждый день / О мщенье помышляю... / И ныне, кажется, мой час настал“ (PušKIN 1975: 351).

Während also Puškins Rusalka als zutiefst zerrüttetes und vom Leben ungerecht behandeltes Wesen mit emotionalem Tiefgang in Erinnerung bleibt, ist auch die von Lermontov in der gleichnamigen Ballade aus dem Jahr 1836 beschriebene Rusalka eine empfindsame Kreatur. Für die Analyse des Musikvideos $Я$ тебя зацелую von „Фабрика“, in dem die Sängerinnen als Rusalki auftreten, ist dieses Gedicht Lermontovs insbesondere deshalb interessant, weil es als das erste Werk gelten kann, in dem die bisher meist schweigsamen Wassernixen von einer Rusalka abgelöst werden, die durch ihren lyrischen, klangreichen Gesang fasziniert. Entgegen dem Stereotyp der Männer mordenden Sirene zeigt sich die Rusalka des jungen Lermontov als erfüllt von unverständlicher Wehmut (,,[п]олна непонятной тоской“, LERMONTOV 1948: 11). Sie versucht nicht, ein männliches Opfer anzulocken, sondern beweint den (nicht von ihr verschuldeten) Tod eines jungen Ritters, der nun ihre Küsse nicht mehr erwidern kann. Nicht nur auf der emotionalen, sondern auch auf der beschreibenden, landschaftlichen Ebene hat dieses Gedicht Lermontovs sehr viel mit demjenigen Puškins aus dem Jahr 1819 gemein abermals befinden wir uns an den mit Schilf bewachsenen Ufern eines Flusses, der Mond scheint und aus den silbrig glänzenden Wellen erhebt sich die in diesem Fall singende Rusalka.

Sieht man sich vor diesem Hintergrund nun zeitgenössische Variationen des Rusalka-Motivs in der Popkultur an, so ist ein Bezug auf die zuvor genannten Quellen und ihre eindeutig romantisch inspirierte Bildsprache (Flussufer, rurale Umgebung, romantische Grundstimmung) evident. Gleichzeitig wird aber genau mit dem Konzept romantischer, unerfüllter Liebe und besonders mit der emotionalen Tiefe, zerrütteten Psyche und wahrhaftig empfundenen Trauer der Rusalki aus der Romantik mehrfach gebrochen. Mithilfe von Satire, Humor und dem Umkehren von geschlechtsdefinierten Machtstrukturen wird der Rusalka-Mythos bei „Фабрика“ im Video Я тебя заиелую (2010) aktualisiert und kommerzialisiert. Dies deshalb, weil die ehemals tragischen Wassernixen der Romantik nun als selbstbestimmte, clevere und attraktive Sängerinnen und Geschäftsfrauen im (s)exploitativen und auf Gewinn ausgerichteten Popsystem der Gegenwart funktionieren müssen, das vorwiegend vom Motto „Sex sells!“ bestimmt wird. Zeitgenössische russische Popmusik ist einerseits sexistisch und oberflächlich - nur junge Frauen mit den gängigen Schönheitsstandards entsprechenden Körpern haben die Chance im Popbusiness Fuß zu fassen; ihre musikalischen Fähigkeiten stehen im Vergleich zu ihren Körpern oft im Hintergrund. Andererseits bieten besonders zeitgenössische Musikvideos gerade im restriktiven Umfeld des heutigen Russland 
auch Chancen für Frauen und eine Möglichkeit, Konzeptionen von Weiblichkeit abseits von häuslicher Mütterlichkeit, wie sie die Matrjoschka verkörpert, in den Medien zu verankern und aktiv zu thematisieren.

Musikvideos (hier als leicht zugängliche, popkulturelle Texte verstanden, die Aufschluss über Konzeptionen von Femininität und Maskulinität geben können), eignen sich besonders gut für die Verhandlung komplexer Inhalte, da sie von einem semiotischen Standpunkt betrachtet „,very complex textual forms“ (PEVERINI 2010: 135) mit „,sehr große[r] Informationsdichte“ (RösING 2003: 19) sind: „They use the expressive potentiality of different languages (visual, musical, verbal) in order to combine the commercial aims of the music business with original aesthetic experiments“" (PEVERINI 2010: 135). Auch Ute Bechdolf sieht Musikvideos im weitesten Sinne des Wortes als kulturellen Text, ,,der mehrere Diskurse hervorbringen und unterstützen kann und dadurch zahlreiche, auch sich widersprechende Lesarten zuläßt" (BECHDOLF 1999: 50). Trotz dieser Möglichkeit, auch Subversives in Musikvideos zu verarbeiten, ist für Bechdolf die „Polysemie der Texte... nicht unendlich“, da durch „spezifische Blickkonstruktionen, direkte ZuschauerInnenAdressierung, subjektive Kamera oder die Montage“ (BEChdolf 1999: 50) im Video immer eine den hegemonialen Ideologien entsprechende Bedeutungsvariante priorisiert respektive dominant erscheint, die auch von Text, Rhythmus und Melodie des Songs unterstützt wird:

Männlichkeit und Weiblichkeit entstehen textuell durch angebotene (und in der Rezeption eingenommene oder abgelehnte) Subjektpositionen. Musikvideos können somit als alltägliche Schauplätze der diskursiven Konstruktion von Geschlecht betrachtet werden - in jedem einzelnen Text wird Geschlecht immer wieder aufs neue verhandelt, Dominantes oder auch Alternatives teilweise akzeptiert oder zurückgewiesen (BECHDOLF 1999: 51).

Wenn wir uns nun also dem Video Я тебя зацелую zuwenden, so wird vielleicht nicht auf den ersten, zumindest aber auf den zweiten Blick klar, dass man die präsentierten Genderzuschreibungen nicht so einfach den beiden entgegengesetzten Polen der Rusalka als Retterin oder aber als Rächerin zuordnen kann. Vielmehr handelt es sich um eine satirische Neuinterpretation der Rusalka, die nicht mehr aufgrund einer traumatisierenden Biographie nach Rache sinnt, sondern komödiantisch auf Männerjagd geht und damit statt Rache an dem sie verstoßenden Liebhaber als sexy Wassernixe Verkaufszahlen im Popbusiness erreichen will.

Entsprechend der Anzahl der Bandmitglieder treten die Rusalki - oder „,rusaločki“" wie sie sich im Songtext ${ }^{19}$ verniedlichend und verharmlosend nennen nun zu dritt auf. Am Ufer des Sees friedlich inmitten einer Auenlandschaft sitzend, erinnern die jungen Frauen mit ihren langen, lockigen Haaren durchaus an die klassischen Rusalki. Sie haben aber ganz offensichtlich gerade in Bezug auf ihre Optik eine Modernisierung durchgemacht: Die jungen Frauen sind nämlich nicht völlig nackt, sondern entsprechend den aktuellen Modetrends gekleidet (die Outfits

${ }^{19}$ Der Songtext ist abrufbar unter: http://megalyrics.ru/lyric/fabrika/ia-tiebia-zatsieluiu.htm. 
bestehen aus knappen, seidigen Negligees, Kniestrümpfen und Pelzmäntelchen); die Haare sind nicht mehr nass - das würde die aufwendig zurecht gedrehte Frisur zerstören - und auch das Make-up wurde dick aufgetragen, wodurch die Protagonistinnen ganz im Gegensatz zu ihren mythologischen Vorgängerinnen in starkem Kontrast zur ländlichen Umgebung stehen, in die sie nicht so recht passen wollen. Die Sängerinnen der russischen Casting-Girlband „Фабрика“, die 2002 beim Showformat „Фабрика звезд“ zweite wurden und seitdem mehrmals die Besetzung wechselten, stellen in ihrem Video zwar Rusalki dar, sind aber weit von den mit der Natur und dem Element Wasser im Einklang lebenden Wesen, die Puškin und Lermontov imaginiert haben mögen, entfernt. Rusalka 2.0 sozusagen.

Durch die Kameraführung, die auf die körperlichen Vorzüge der Sängerinnen fokussiert, scheinen die Frauen als Objekte für männliche Blicke inszeniert zu sein, denen kein aktiver Handlungsspielraum zusteht. Auch der zu Beginn vorgetragene Text lässt vorerst nichts Gegenteiliges erahnen: „Как на берегу озера тайком / Загорали русалочки втроем / Наболтали русалки о любви“, doch sowohl die Beschreibung als Rusalki als auch die vierte Zeile „Кто кого заманил в свои сети“ können bereits als Vorwarnung für den weiteren Verlauf des Videos gelten. Schon bald stellt sich nämlich heraus, dass die scheinbare Handlungsunfähigkeit ein Trugschluss ist und sich die vermeintlichen Wassernixen nicht nur optisch weiterentwickelt haben. Im 21. Jahrhundert ist nun keine Rede mehr von empfindsamen, traumatisierten jungen Frauen, die des Nachts wegen ihrer durch Selbstmord ausgelösten Transformation zur Rusalka ihren ehemaligen Liebhabern bzw. stellvertretend allen Vertretern des männlichen Geschlechts auflauern. Vielmehr spielt das Video Я тебя заиелую am helllichten Tag - die „rusaločki“, die immer die Kontrolle über die Ereignisse behalten, scheinen sich in Videospiel- und SlapstickManier einen Spaß daraus zu machen, vorbeikommende Jünglinge zu jagen, sich mit ihnen zu vergnügen und ihnen Gewalt anzutun. Die Entwicklung vom Küssen und Umarmen bis hin zum Ertränken dauert auch auf der Ebene des Songtextes nicht lang: „Я тебя зацелую, обниму сильно-сильно / Я тебя заколдую до утра долго-долго / Я тебя зацелую и скажу нежно-нежно / Если я полюбила, значит ты мой навечно. // Не смотри мне в глаза, заворожу / Ой, да не убежать, когда гляжу / Я во взгляде его сама тону / Ох, утянет любовь меня ко дну“.

Auch was die Auswahl der Opfer betrifft, kommt nun bei weitem nicht mehr jeder in Frage. In der bunten, lauten Popwelt der Reichen und Schönen hätte ein Puškin'scher alter Mönch mit grauem Bart zu wenig Sexappeal, um von den mörderischen Damen auch nur eines Blickes gewürdigt zu werden - nun werden Bauernburschen in Männermodel-Optik mit verschwindend geringem Intelligenzquotienten und Reaktionsvermögen von den Performerinnen mit viel komödiantischem Talent für Hilfsarbeiten eingeteilt, auf die Schaufel genommen, verdroschen (bei 01:32 erfolgt ein gezielt ausgeführter Faustschlag einer der scheinbar passiven Schönen), gefesselt und ertränkt - die Entsorgung der vermeintlich angefallenen Leiche wird von einem klar hörbaren platschenden Geräusch, wie man es aus Comics kennt, begleitet. Doch bevor es dazu kommt, werden die potentiellen Opfer ausgiebig begutachtet und mit Bedacht ausgewählt: „Чу! По берегу парень да 
молодой / Ой, девчонки, хорошенький он такой / Ой, подружки, влюбилася, не могу / Ой, девчата, хочу я его, хочу“.

Unverblümt und selbstbewusst geäußertes sexuelles Begehren seitens junger Frauen (,,хочу я его, хочу“) ist in der zeitgenössischen, von traditionellen Rollenbildern geprägten russischen (Pop-)Kultur keine Selbstverständlichkeit - selbst wenn dieses Begehren eingebettet in den kulturgeschichtlichen Hintergrund der immer schon mit Sexualität konnotierten Rusalki präsentiert wird. Auch die eindeutig feminin bestimmte Machtverteilung zwischen den Geschlechtern, die im Video im Vergleich zur zeitgenössischen russischen Gesellschaft komplett umgedreht wird, ist nicht nur auf der visuellen Ebene präsent (Gewalt an Männern, bewaffnete Frauen), sondern auch im Text: „Если я полюбила, значит ты мой навечно“. Wen die Rusalka einmal als Opfer auserkoren hat, der muss sich für immer deren Allmacht fügen. Flucht, Widerspruch oder Protest bleiben aussichtslos.

Dabei wird nicht nur die weitgehende Ohnmacht der Frau in der heutigen russischen Gesellschaft konterkariert, sondern auch die typische nach dem Geschlecht festgelegte Täter-Opfer-Rollenverteilung umgekehrt, woraus humoristisches Kapital geschlagen wird. Auch wenn hier also ein durchaus überraschendes Aufbrechen klassisch tradierter Geschlechterrollen hin zu einer aktiven bis humoristischaggressiven Frauenrolle festzustellen ist, so ist die bloße Umkehrung von Unterdrückungsstrukturen und Gewaltphantasien zugunsten der Frauen - auch wenn sie ironisch erfolgt - noch lange kein Grund zur Freude über erlangte Gleichberechtigung. Aufgrund der Tatsache, dass momentan im zeitgenössischen russischsprachigen Pop eine Vielzahl von Girlbands (vgl. insbesondere die aus der Ukraine stammende Band „NikitA“) mit Videos erfolgreich ist, die Gewaltakte an Männern beinhalten, scheint es eher angebracht, die von Eliot Borenstein konstatierte Krise der Männlichkeit im russischsprachigen Raum zu bestätigen (BORENSTEIN 2008: 45, vgl. dazu LECHNER 2014: 98-118). Während also die aktualisierte Figur der Rusalka die Möglichkeit bietet, neue und aktive Frauenbilder, die selbstbewusst mit ihrer Sexualität umgehen, im Mainstream zu verorten, ist die Komponente der an Männern ausgeübten Gewalt wiederum ein Rückschritt für ein gleichberechtigtes Miteinander der Geschlechter.

\section{„Alkonost“": Русалка}

Das dritte und letzte Beispiel, von dem in diesem Beitrag die Rede sein soll, ist zwar (bis in seinen Titel Русалка hinein) von analogen Zuschreibungen an die Rusalka geprägt, wie dies bereits im Falle von Doda Elektrodas Song Electrode und der Nummer Я тебя зацелую der russischen Girlband „Фабрика“ sowie der dazugehörigen Musikvideos konstatiert werden konnte; allerdings erfolgen diese Zuschreibungen nun sowohl auf musikalischer als auch auf textueller Ebene in deutlich modifiziertem Gewand, was seinerseits wiederum die Wandelbarkeit und prinzipielle Dynamik des Rusalka-Motivs belegt, das unter Beibehaltung seiner zentralen semantischen Dimensionen offensichtlich in ganz unterschiedliche stilistische Kontexte eingespannt werden kann. Bei Doda Elektroda und „Фабрика“ 
geschah dies entsprechend der medial vermittelten Weiblichkeitsbilder der Popkultur primär im Zeichen der verführerischen schönen Frau, die (mindestens im Falle von Я тебя заиелую) den Männern zum Verhängnis wird. Einen im Unterschied dazu etwas anders gelagerten Umgang mit dem Motiv der Wasserfrau bietet der Song Русалка der russischen Folk-Metal-Band „Alkonost“" aus dem Jahr 2014 (eine akustische Version davon wurde ein Jahr darauf veröffentlicht). ${ }^{20}$ Die 1995 in der Stadt Naberežnye Čelny in Tatarstan gegründete Band thematisiert in ihren Nummern immer wieder Motive des russischen Volksglaubens (wie eben auch den Paradiesvogel Alkonost) ${ }^{21}$ in einer affirmativen Weise, von daher scheint die Hinwendung der Gruppe zu einem derart prominenten Folkloremotiv wie der Wasserfrau durchaus naheliegend; die dahinterstehende Intention ist bei „Alkonost" anders als bei Doda Elektroda und „Фабрика“ (also im polnischen und russischen Girl-Pop) wohl weniger in der erotischen Aufladung des Motivs oder im Versuch zu suchen, dem Motiv neue Facetten von Weiblichkeit abzugewinnen, als in den mythologischen Dimensionen der Rusalka.

Was den Song der russischen Band dessen ungeachtet mit den beiden zuvor analysierten Nummern auf performativer Ebene vergleichbar macht, ist der Umstand, dass er ebenfalls von einer Frau gesungen wird: „Alkonost“ zählt nämlich zu jenen Metal-Bands, deren Songs von einer Sängerin mit einer ausgebildeten Stimme präsentiert werden, die von ihrer Modulation her bisweilen in Richtung des klassischen Fachs geht und auf diese Weise verschiedene Segmente der Musikkultur miteinander verbindet. Im Falle von „Alkonost“" wurde dieser spezifische Stil durch die Sängerin Alena Pelevina in die Band eingebracht und später dann in modifizierter Manier von Ksenija Pobužanskaja weitergeführt. Pobužanskaja ist dann auch jene Sängerin, die der Rusalka in der entsprechenden Nummer ihre Stimme verleiht, was sich auf zweierlei Weise auswirkt: Einmal erwächst der für Metal-Bands allgemein kennzeichnenden Hypostasierung des Männlichen (vgl. BRILL 2009) ein weibliches Gegengewicht, sodass die Geschlechterrollen hier tatsächlich - wie Diedrich Diederichsen konstatierte - ins sogenannte „Rollen“ kommen (DieDERICHSEN 1996: 44) und sich im performativen Akt des Songs in der Interaktion der verschiedenen Stimmen neu gewichten ${ }^{22}$ - der typische Männlichkeitskult des Metal spricht durch die Sängerin mit einer zu diesem in Widerspruch stehenden weiblichen Stimme, was die Konstitution eindeutiger Gender-Zuschreibungen von vorneherein blockiert.

Zusätzlich dazu ist die mythologische Gestalt der Wasserfrau in „Alkonosts“ heidnisch-nationaler russischer Metal-Welt nicht nur über den Gesang von Ksenija Pobužanskaja anwesend - so wie in den beiden zuvor thematisierten Songtexten Doda Elektrodas und der Girlband „Фабрика“ sowie in Puškins Drama ist die

${ }^{20}$ https://en.wikipedia.org/wiki/Alkonost_(band) bzw. https://ru.wikipedia.org/wiki/Alkonost

${ }^{21}$ Zum Fabelwesen Alkonost vgl. BelovA 1993 bzw. BELOVA-KVLIVIDZE 2001.

${ }^{22} \mathrm{Vgl}$. dazu das Agieren der Bandmitglieder von „Alkonost“" während des ,Folk Summer Fest“" direkt auf der Bühne, https://www.youtube.com/watch?v=OFIIF4ZiAyY - Ksenija Pobužanskaja ist es in ihrer Eigenschaft als Sängerin hier vorbehalten, dem Konzertpublikum den Auftritt ihrer Band anzukündigen (die Nummer Русалка ebendort ab 06:26). 
Rusalka auch im Songtext von „Alkonost“ direkt mit einer weiblichen Stimme präsent. Sie wird also nicht über die Form (männlicher) Fremdzuschreibung von außen (wie in Puškins Gedicht), sondern als weibliche Selbstzuschreibung konstituiert und kommt so direkt zu Wort. Die Wasserfrau der russischen Band vermag für sich selbst zu sprechen und von daher in der ersten Person Singular für sich selbst Identität zu beanspruchen: In den Strophen II und III des Songtextes geschieht dies in der jeweils ersten, analog gehaltenen Zeile „Там мой дом“"23 in Form des Possessivpronomens (analog auch in Strophe VII „В кой пришел мой тать!“), ehe in der letzten Zeile von Strophe V „Надо мною синеокой“ das Personalpronomen als weiterer grammatikalischer Schritt in Richtung Konstituierung des weiblichen Subjekts hinzutritt. Abgeschlossen wird dieser Prozess dann in der unten zitierten, letzten Strophe des Textes: „Цветет всех нежней / белая лилия / Светлых вод ясней / Белая лилия / Как я тебя ждала... / Белая лилия / В омут вод звала / Белая лилия / Крепко обняла... / Белая лилия / В омут увлекла / Белая лилия / Гладь воды светла / Белая лилия / Где цветет, нежна / Белая лилия....“.

Hier finden sich in der fünften Zeile „Как я тебя ждала...“ die Rusalka und ihr direkt adressierter Geliebter unmittelbar zueinander in Relation gestellt. Unter diesem Gesichtspunkt könnte für den Songtext eine sukzessiv Kontur gewinnende Subjektkonstitution der titelgebenden Rusalka konstatiert werden. In dieser zwölften und letzten Strophe des Textes kommt freilich nicht nur die Rusalka (mindestens auf syntaktischer Ebene) schließlich zu sich selbst und rückt in die Position des von sich selber sprechenden Subjekts ein, diese Passage des Lieds erweist sich in gleich mehrfacher Hinsicht als besonders relevant: Erstens ist sie mit gezählten sechzehn Zeilen schon rein quantitativ bei weitem umfangreicher als die vorangegangenen Strophen, die zumeist aus lediglich vier Zeilen bestehen; die Funktion als den Song beschließender Textblock ist hier unverkennbar. Zusätzlich dazu erhält das Motiv der Rusalka hier sowohl auf der syntagmatischen als auch auf der paradigmatisch-kontextuellen Achse des Textes seine entscheidenden Konturen. In den drei Zeilen „В омут вод звала / Крепко обняла... / В омут увлекла“ entfaltet sich das traditionelle Sujet der Wasserfrau, die den Mann zu sich ins Wasser lockt, ihn umarmt und schließlich in ihren Bereich in die Tiefe herabzieht, was den Songtext wiederum an die entsprechende Literarisierung des Rusalka-Motivs etwa im Gedicht von Puškin anschließt.

Auf der paradigmatisch-kontextuellen Achse wiederum generiert das rezitativartig in jeder zweiten Zeile wiederholte Motiv der weißen Lilie einen Konnex zum Motivsystem des russischen Frühsymbolismus der Jahrhundertwende, der das Motiv der Wasserfrau von den kanonisierten Autoren der Romantik wie Puškin oder Lermontov übernahm, aber entsprechend umcodierte. Die Referenz in Richtung Frühsymbolismus am Textende wird durch entsprechende motivische Verweise am Anfang des Liedtextes ergänzt, vgl. Strophe I: „Свеж, чист / Омут озеpa зеркальный, / Тих лист / Камыша под брегом дальним“ - die (glatte, verlockende) Oberfläche der Untiefe funktioniert hier in ihrer Bewegungslosigkeit als

${ }^{23}$ Русалка (текст песни группы Alkonost), http://samlib.ru/m/merke_m/mermaid.shtml. 
Medium der Spiegelung und damit als Problematisierung stabil gesetzter Identitäten, die Schilflandschaft wiederum als Index für den Übergang und die Grenze zwischen Land und Wasser als zwei entgegengesetzten semiotischen Sphären, zwischen denen sich die Rusalka bewegt. ${ }^{24}$ Auch der Schatten in Strophe VII („Пала тень / На вод лазоревую гладь“) und die Stille in Strophe X (,Там мой дом - / Тишь-покой хранит“) ließen sich ohne Probleme in das Motivsystem des russischen Frühsymbolismus hineinstellen: der Schatten analog zum Spiegelmotiv im Zeichen der Problematisierung von Identität über die Polarität von Urbild und Abbild, die Stille wiederum über die Problematisierung von Kommunikation bzw. die gänzliche A-Kommunikation. ${ }^{25}$

Über dieses Bündel an wichtigen poetischen Motiven schließt der Songtext der Metal-Band „Alkonost“" an mehrere Gedichte des russischen Frühsymbolismus an, die analog die Wasserfrau im Titel tragen, die Rusalka in das zwischen Femme fatale und Femme fragile pendelnde Frauenbild in der Kunst der Jahrhundertwende transformieren und ihr auf diese Weise neue ästhetische Relevanz zusprechen. Ein frühes Beispiel bietet diesbezüglich Konstantin Dmitrievič Bal'monts Gedicht Она как русалка, das 1898 als Teil des Zyklus Ветер с моря in der Sammlung Тишина veröffentlicht wurde. Neben dem Titel des Gedichtbandes, der das zuvor angesprochene Fehlen einer Kommunikation evoziert (vgl. die Stille im Songtext von „Alkonost"), bietet auch Bal'monts Gedicht selbst mehrere Übereinstimmungen mit dem Songtext auf motivischer Ebene: So erscheint die geschilderte Frau seltsam-bleich - „странно-бледна“ (BAL'MONT 1969: 125) (6 $^{26}$ und korrespondiert auf diese Weise mit der weißen Lilie in ihrer Funktion als Zentralmotiv der $P y$ салка von „Alkonost". Die Untiefe des Songtextes („омут“) wiederum findet sich in Bal'monts Gedicht in die grünen Augen der mit einer Rusalka verglichenen Frau verlagert: „В зеленых глазах у нее глубина - холодна“. In der Mittelstrophe des Gedichts wird die Umarmung der Wasserfrau, die im Songtext als Ereignis in der Vergangenheit erwähnt wird („Крепко обняла“), als zukünftige Verheißung präsentiert („Приди - и она обоймет, заласкает тебя“), ehe in der letzten Strophe ganz analog zum Titel des gesamten Bandes Тишина das Schweigen der Wasserfrau unter dem Zeichen des Mondes (oder präziser: der Mondin) Erwähnung findet („,И будет молчать под луной“). Bereits dieses relativ frühe Rusalka-Gedicht des russischen Symbolismus weist also mehrere deutliche motivische Korrespondenzen zum Songtext von „Alkonost“" auf.

Einige Jahre später kam Bal'mont dann erneut auf das Motiv der Wasserfrau zurück und nahm in seinen Band Будем как солние 1903 auch ein zweistrophiges Gedicht mit dem Titel Русалка auf, in dem die Wasserfrau analog zum Songtext

\footnotetext{
${ }^{24}$ In den Gedichten des russischen Frühsymbolismus findet sich das Motiv des Schilfs mehrfach im Zeichen des Übergangs zwischen den einzelnen Sphären wie auch im Zeichen der a-kommunikativen Flüstersprache, in der sich das Schilf(-rohr) artikuliert (vgl. HANSEN-LövE 1989: 207-209).

${ }^{25}$ Zum Motiv des Schattens in der Lyrik des russischen Frühsymbolismus vgl. HANSEN-LÖvE 1989: 253-264, zu den diversen Manifestationen der problematisierten Kommunikation vgl. HANSENLöVE 1989: 187-201.

${ }^{26}$ Alle weiteren zitierten Passagen aus dem Gedicht finden sich auf derselben Seite.
} 
direkt zu Wort kommt. Hier fungieren die Blässe und die Existenz unter Wasser anders als noch 1898 nicht als Fremd-, sondern als Selbstzuschreibung der Rusalka. Der Umstand, dass dies mit analogen Begriffen geschieht, zeigt Bal'monts bisweilen inflationäre Kombinatorik auf motivischer Ebene. Hier spricht die Wasserfrau nun den Mann direkt an und erklärt: ,До тебя я была холодна и бледна. / Я - с глубокого, тихого, темного дна“ (BAL'MONT 1969: 250). ${ }^{27}$ Zwei Jahre danach griff Bal'mont in seinem Band Фейные сказки. Детские песенки 1905 im Gedicht Русалочка auf eine verniedlichte Wasserfrau eben im Format des Kinderlieds zurück. Diese besinnt sich gemeinsam mit ihren Gefährtinnen auf ihre ursprünglichen Aktivitäten, das Kichern und das Kitzeln: „Они меня щекочут, / [...] А им-то что, хохочут“ (BAL'моNт 1980: 226). Im Unterschied zum Songtext von Русалка firmiert hier nicht die Wasserfrau, sondern ihr männliches Opfer als lyrisches Subjekt, das entsprechend der Stilisierung in Richtung Kinderlied von den Rusalki hier freilich nicht in das Wasser gelockt, sondern lediglich mit leichten Schlägen traktiert und schließlich nass und beschämt zurückgelassen wird (BAL'MONT 1980: 227).

Ebenfalls im Jahre 1905 veröffentlichte Nikolaj Gumilev seinen ersten Gedichtband Путь конквистадоров, der noch weitgehend im Kontext des russischen Symbolismus steht. Dies belegt etwa der Titel Bысоты и бездны eines Zyklus, der ein Einzelgedicht mit dem Titel Русалка enthält. Hier wird die Wasserfrau vom lyrischen Subjekt als ,дева-ундина“ bezeichnet und so ein anderes Begriffsregister eröffnet, das zu „Alkonosts“ forcierter Hinwendung zur russischen Mythologie in Kontrast steht (GUMILEV 1991: 38). Wichtiger scheinen demgegenüber aber die beiden abschließenden Zeilen des Gedichts, in dem sich das lyrische Subjekt nicht in einen Gegensatz zur geliebten Rusalka stellt, sondern ganz im Gegenteil seine Liebe zur Wasserfrau mit der analogen Herkunft aus den Wellen des Meeres erklärt: „Потому что я сам из пучины, / Из бездонной пучины морской““ (GuMILEV 1991: 38). Gumilev behält hier die symbolistische Motivik des Abgrunds sowohl im Titel seines Zyklus Bысоты и бездны als auch im Attribut „бездонной““ direkt im Einzeltext bei. ${ }^{28}$ Gleichzeitig ersetzt er aber den traditionellen elementaren Gegensatz zwischen der Frau aus dem Wasser und dem in ihren eigenen Bereich gelockten Mann, der den festen Grund unter seinen Füßen verliert und eine

${ }^{27}$ In Bal'monts Gedicht Я ласкал ее долго, ласкал до утра... firmiert das Leben in der Tiefe als kollektive Selbstzuschreibung der Rusalki überhaupt, wenn eine der Wasserfrauen singt: „Мы с глубокого дна, и у той глубины / Много дев“ (BAL’мONT 1969: 251). Rainer Georg Grübel erwähnt zum Gedicht Русалка, dass Bal'mont hier (analog zum Songtext von „Alkonost“) die Wasserfrau unmittelbar zu Wort kommen lässt und damit ,die Stufe höchster Empathie gerade dem mythischen Wesen“ gewährt (GRÜBEL 1995: 144). Maria Deppermann geht in ihrem Beitrag zu den Wasserfrauen nur knapp auf Bal'monts Gedichte ein und bemerkt: „Gemäß der Vorherrschaft der Linie im floral-vegetativen Stilsystem des Jugendstils nehmen Lianen, Wasserpflanzen, Nymphen, Najaden - wie wir sie auch in der Malerei finden - einen großen Raum in seiner frühen Dichtung ein“" (DEPPERMANN 2006: 178); zum Rusalka-Motiv in Bal'monts früher Lyrik vgl. SCHNEIDER 1970: 151f.

${ }^{28}$ Zum Motiv des Abgrunds in der Lyrik des russischen Frühsymbolismus vgl. HANSEN-LÖVE 1989: 147-149; zur Positionierung von Gumilevs Werk im Kontext des Symbolismus vgl. weiter KLING 1995. 
für ihn selbst verhängnisvolle Grenzüberschreitung in ein anderes Element vollzieht, durch eine gegenteilige, verbindende maritime Genealogie (zu diesem Gedicht Gumilevs vgl. GRÜBEL 1995: 148-150).

Selbst wenn damit noch keine Vorwegnahme des Akmeismus, den Gumilev acht Jahre später mit seinem Manifest Наследие символизма и акмеизм mitbegründen sollte, verbunden sein mag, so zeigt sich in diesem neuen Frauenbild doch ein deutliches Abrücken von der frühsymbolistischen Femme fatale, das Bal'mont um die Jahrhundertwende mit der Wasserfrau verband, auf das aber auch Valerij Jakovlevič Brjusov mindestens in Ansätzen noch 1907 in seinem ebenfalls mit Русалка überschriebenen, 1909 in der Sammlung Все напевы veröffentlichten Gedicht zurückgriff (ursprünglich sollte das Gedicht mit Наяда betitelt werden, was neben Gumilevs Undine eine weitere alternative Terminologie ins Spiel gebracht hätte, Brjusov entschied sich letztlich dann aber mit Русалка für einen anderen Titel und stellte den eigenen Text so bewusst in die Tradition der RusalkaDichtungen der russischen Literatur, vgl. BRJusov 1973: 645). Von besonderer motivischer Relevanz sind hier Anfang und Ende des sechsstrophigen Gedichts, da diese einen Konnex zur Русалка von „Alkonost“ eröffnen: In der ersten Strophe des Songtextes wird in den Zeilen 3 und 4 das Schilf als Element der Wasserwelt eingeführt („Тих лист / Камыша под брегом дальним“), in der Русалка Brjusovs wird das Schilfrohr in Strophe I mit der für den Frühsymbolismus kennzeichnenden pendelnden und daher nicht produktiven Bewegung (,зыбким“) verbunden und in erotischer Funktion als spärliche Kleidung der Wasserfrau ins Spiel gebracht, die deren Brüste und Schoß bedeckt („Она [русалка], свои скрывая груди / И лоно зыбким тростником““ [BRJUSOv 1973: 502]) - von dieser sexualisierten Darstellung der Rusalka ergibt sich ein direkter Konnex zu popkulturellen Akteurinnen wie Doda Elektroda oder Eva Bušmina in ihrer jeweiligen Interpretation der Wasserfrau. Mit dem Motiv des Schilfs wird das Gedicht Brjusovs dann in Strophe VI unmittelbar abgeschlossen: „Но, видя проходящих парней, / Вечеровой порой, в тиши, / Еще нежней, еще коварней / Смеялась, зыбля камыши“" (BRJUSOv 1973: 503).

Hier sind gleich zwei unmittelbare motivische Parallelen zum Songtext bis in die jeweilige Lautung zu konstatieren, was wiederum belegt, dass die popkulturelle Rusalka der russischen Metal-Band zum entsprechenden Motiv in der russischen Literatur der Jahrhundertwende in Verbindung steht. Die Stille (,тишь“) findet sich in Songtext und Gedicht, hier ist sie a-kommunikatives Merkmal für die Übergangszeit des Abends, das Schilf (diesmal analog zum Songtext als „Камыши“) wird in der Schlusszeile von der Wasserfrau selbst in jene pendelnde Bewegung versetzt, die bereits am Anfang des Textes aufgerufen wird. An beiden semantisch besonders exponierten Positionen des Gedichts werden also das pendelnde Schilf und die Rusalka in einen direkten Konnex zueinander gestellt. Das Moment der A-Kommunikation überwiegt hier die motivischen Restbestände der Femme fatale: Die Rusalka lächelt zwar zärtlich und tückisch aus ihrem Schilfversteck heraus, scheint die vorbeigehenden Burschen jedoch eher zu beobachten als zu sich ins Wasser zu rufen. 
An anderen Stellen des Gedichts beobachtet die Rusalka die Menschen heimlich aus dem Fluss heraus (BRJusov 1973: 502) oder ist für diese unter dem Wasser kaum sichtbar (BRJUSOv 1973: 503); der Versuch, mit der im Fluss spielenden Kinderschar in Kontakt zu treten, schlägt fehlt, da die Rusalka der Menschenwelt prinzipiell fremd ist und sich folgerichtig in einer unverständlichen Sprache artikuliert (,откликаясь / на непонятном языке“ [BRJUSOv 1973: 503]). Brjusov ist ganz offensichtlich daran gelegen, die existenziellen Bereiche von Wasserfrau und Menschen als einander grundlegend fremd und unverständlich zu markieren - die Gebete der Menschen dringen von daher auch als geheimnisvolle Beschwörungen in ihre Unterwasserwelt (BRJusov 1973: 503). Diese für die Sprachskepsis der Moderne nicht untypische Positionierung fehlt im Songtext, sie ist mit dem MetalGenre ganz offenbar schlicht nicht kompatibel. Folgerichtig bleibt die verhängnisvolle Kommunikationssituation im Songtext entsprechend der Tradition aufrecht, die Rusalka hat hier in der zitierten umfänglichen Schlussstrophe den Geliebten zu sich in die Tiefe des Wassers gerufen („,В омут вод звала“). ${ }^{29}$

Auch wenn sich Sprechen und Schweigen der Wasserfrau im Song von „Alkonost" nicht direkt problematisiert finden, bleibt die über die zuvor erwähnten Motive generierte Nähe zwischen Liedtext und frühsymbolistischer Lyrik dennoch bestehen. Einen deutlichen Hinweis in diese Richtung bietet auch die bildliche Ebene, die der musikalischen im Internet hinzugefügt ist: Zur Nummer Русалка existiert anders als zu Doda Elektrodas Song Electrode und zu Я тебя защелую von „Фабрика“ zwar kein eigenes Musikvideo, mindestens jedoch eine aufwendig gestaltete Illustration, in der die Rusalka eindeutig in die Bildsprache von Impressionismus und Symbolismus integriert wird. ${ }^{30}$ Die Wasserfrau wird hier von Ksenija Pobužanskaja selbst verkörpert, die Sängerin steht in einem weißen Kleid während einer Vollmondnacht im Wasser, das genau von jenen weißen Wasserlilien bedeckt ist, die im Songtext intensiv evoziert werden. Die Gestalt der Rusalka wird deutlich wahrnehmbar im Wasser gespiegelt, was die zweite Zeile des Songtextes „Омут озера зеркальный“ entsprechend veranschaulicht. Sind all diese optischen Signale im Referenzrahmen der Bildenden Kunst der Moderne situiert, ${ }^{31}$ so lässt sich der in altrussischen Lettern gehaltene Songtitel Русалка als bewusst gesetzte ideologische Strategie zur Identitätsstiftung in einem betont russisch-nationalen Kontext interpretieren, der den globalen Strukturen der Popkultur ein radikal alternatives Narrativ entgegenhält.

Die altrussischen Lettern firmieren in der russischen Metal-Szene ganz offensichtlich als Erkennungszeichen für eine ähnliche ideologische Ausrichtung, die Geschichte und Kultur der eigenen Nation privilegiert setzt und diesen besonderen Status auf diese Weise manifestieren möchte. Ein entsprechendes, zur Русалка von „Alkonost“ analoges Beispiel bietet etwa das Cover des Albums Вольницей

\footnotetext{
${ }^{29}$ Rainer Georg Grübel sieht in der doppelten Attributierung des Lächelns der Rusalka die Andeutung eines ethischen Charakters gegeben (GRÜBEL 1995: 146).

${ }^{30} \mathrm{https}: / / \mathrm{www}$. youtube.com/watch? $=\mathrm{MSq} 0 \mathrm{MoS} 4 \mathrm{t} 18$

${ }^{31}$ Vgl. dazu den Abschnitt Undinen-Zauber. Zum Frauenbild des Jugendstils (HERMAND 1989: 469-494) bzw. den Abschnitt Jugendstil-Nixen (Отто 2001: 128-149).
} 
в просинь ночей (2005) der russischen Pagan-Metal-Band „Темнозорь““, 32 auf dem der martialische Titel in analogen Lettern gehalten ist. ${ }^{33}$ Weiter geht auch die Covergestaltung der Pagan-Metal-Band „Аркона“, die mit Marija Archipova ähnlich wie „Alkonost“" ebenfalls eine Sängerin aufweist, in eine ganz ähnliche Richtung, ${ }^{34}$ analog dazu das Cover des Debütalbums Кудель белоснежного льна $(2008)^{35} \mathrm{der}$ russischen Folk-Metal-Band „Калевала“, bei der mit Ksenija Markevič wiederum eine Sängerin aktiv ist. Alle diese Alben bzw. einzelnen Nummern generieren zusammen eine mythologisch überhöhte Vorstellung einer vorchristlich-heidnischen Rus', die nicht von der orthodoxen Kirche, sondern der slawischen Götterwelt geprägt ist und in die sich die Gestalt der Wasserfrau gerade in ihrer Wandelbarkeit und erotischen Ausstrahlung ohne Probleme einpassen lässt. Gleichzeitig partizipieren die drei in diesem Beitrag präsentierten, stilistisch durchaus unterschiedlichen Songs an der polnischen und russischen Literatur im Kontext von Romantik und Moderne - an einem Ensemble von Texten also, das von hoch kanonisierten Autoren wie Mickiewicz, Puškin oder Lermontov miterstellt wurde.

Über diese diachrone literarische Linie aus dem 19. Jahrhundert heraus wird symbolisches Kapital akkumuliert, das die Popkultur im Zuge der von Diederichsen hervorgehobenen popkulturellen Strategie einer stetigen Neucodierung dazu nutzt, um es in der Gestalt der Rusalka nochmals zu sexualisieren und an konkrete weibliche Popstars wie Doda Elektroda oder die Mitglieder von „Фабрика“ anzubinden. Der Umstand, dass die Wasserfrau selbst eine fluide, transgressive Existenz zwischen den Elementen Wasser und Erde führt, erleichtert naturgemäß Zuschreibungen dieser Art in erheblichem Ausmaß (die prinzipielle Wandelbarkeit des Rusalka-Motivs zeigt sich ja bereits im Bereich der Literatur selbst, wie anhand der Texte von Bal'mont, Brjusov und Gumilev dargelegt wurde, die bereits eine deutlich andere Variante der Wasserfrau generieren als die Texte der Romantik). Auf diese Weise ist die Rusalka dazu in der Lage, verschiedene kulturelle Segmente zueinander in Relation zu stellen (vgl. etwa das Motiv des Schilfs im Gedicht von Brjusov und im Songtext von ,Alkonost“) und so für sich selbst immer wieder neue Bedeutungen anzunehmen. Letztere mögen aus dem Blickwinkel der Hochkultur heraus vielleicht als abwegig erscheinen, eröffnen de facto aber neue Modi kultureller Verknüpfung und hebeln auf diese Weise das (freilich nicht mehr ganz aktuelle) Verdikt T. Adornos gegen ein Zusammenzwingen von hoher und niederer Kunst durch die Kulturindustrie aus (ADORNo 2013: 16). Die Wasserfrau

${ }^{32}$ Die Relevanz der Rusalka reicht in der Metal-Subkultur freilich über einen engeren russischen Bereich hinaus, wie der ebenfalls mit Rusalka betitelte Song der kroatischen Folk-Metal-Band „Stribog“" von deren Album U okovima vječnosti (2010) belegt, vgl. https://www.youtube.com/watch ?v=uMNegVAWp5M bzw. http://www.songlyrics.com/stribog/rusalka-lyrics/.

${ }^{33} \mathrm{Vgl}$. https://www.youtube.com/watch?v=CxMjspfU8Xo. Zur Band selbst und den Kontroversen um ihre umstrittenen politischen Positionen vgl. https://de.wikipedia.org/wiki/Temnozor. Zum Phänomen des politisch rechtsgerichteten Metal vgl. auch DoRNBUSCH-KILLGUSS 2005, wo entsprechende Bands aus Osteuropa mindestens am Rande erwähnt werden.

${ }^{34} \mathrm{https}$ ://en.wikipedia.org/wiki/Arkona_(band)

${ }^{35} \mathrm{https}: / /$ ru.wikipedia.org/wiki/Кудель_белоснежного_льна 
vermag sich in beiden von Adorno noch strikt voneinander geschiedenen Bereichen frei zu bewegen, ihre Verführungskraft scheint unter diesem Gesichtspunkt weniger eine Angelegenheit der Erotik als eine der Semantik zu sein.

\section{Literatur}

Adorno 2013 = Adorno T. W. Résumé über Kulturindustrie. In: Goer Ch., Greif S., JACKE Ch. (Hrsg.) Texte zur Theorie des Pop. Stuttgart, 2013. 16-22.

BAL'MONT 1969 = БАЛьмонт К. Д. Стихотворения. Ленинград, 1969.

BAL'mont 1980 = БАльмонт К. Д. Избранное. Стихотворения. Переводы. Статьи. Москва, 1980.

BeCHDOLF 1999 = BeCHDOLF U. Puzzling Gender. Re-und De-Konstruktion von Geschlechterverhältnissen im und beim Musikfernsehen. Weinheim, 1999.

Belova 1993 = Белова О. В. О чудесной птице алконост. Русская речь 1993/1:113-117.

Belova-Kvlividze 2001 = БеЛОВА О. В., КвлиВидзЕ Н. В. Алконост. В кн.: Православная энииклопедия. Т. 2. Москва, 2001. 25. http://www.pravenc.ru/text/114518.html.

BorensteIn 2008 = BORENSTEIN E. Overkill. Sex and Violence in Contemporary Russian Popular Culture. Ithaca, 2008.

BRILL 2009 = BRILL D. „Black Metal ist Krieg“. Die mythische Rekonstruktion martialischer „weißer“ Männlichkeit in subkulturellen Musikszenen. In: KAUER K. (Hrsg.) Pop und Männlichkeit. Zwei Phänomene in prekärer Wechselwirkung? Berlin, 2009. 181-204.

BrJusov 1973 = Брюсов В. Собрание сочинений в 7 томах. Т. 1. Москва, 1973.

ButZer-JACOB 2012 = ButZer G., JACOB J. (Hrsg.) Metzler Lexikon literarischer Symbole. Stuttgart, 2012.

DepPermann $2006=$ Deppermann M. Rusalka - Nixe der Slawen. Zur Imago der nicht kanonisierten Frau in der russischen Literatur von der Romantik zur Moderne. In: EIcKMEYER J., SOPPA S. (Hrsg.) Umarmung und Wellenspiel. Variationen über die Wasserfrau. Overath, 2006. 151-197.

DieDERICHSEN 1996 = DieDERICHSEN D. Pop - deskriptiv, normativ, emphatisch. In: HARTGes M., LÜDKe M., Schmidt D. (Hrsg.) Pop, Technik, Poesie. Die nächste Generation. Reinbek, 1996. 36-44.

Dornbusch-Killguss 2005 = Dornbusch Ch., Killguss H.-P. Unheilige Allianzen. Black Metal zwischen Satanismus, Heidentum und Neonazismus. Münster, 2005.

EickMEYer-Soppa 2006 = EicKMeyer J., Soppa S. Vorwort. In: EickMeyer J., Soppa S. (Hrsg.) Umarmung und Wellenspiel. Variationen über die Wasserfrau. Overath, 2006. $7-15$.

FABRE 1956 = FABRe J. Mickiewicz a romantyzm europejski. In: Hagemejer H. (Hrsg.) Adam Mickiewicz 1798-1855 w stulecie śmierci. Warszawa, 1956. 51-74.

FiJAŁKOWSKi 2014 = FuJAŁKOWSKI M. Rusałki - słowiańskie ondyny. Acta Philologica 45. Warszawa, 2014. 219-227. http://acta.neofilologia.uw.edu.pl/archiwum/acta45.pdf.

GARSTENAUER $2010=$ GARSTENAUER T. Geschlechterforschung in Moskau. Expertise, Aktivismus und Akademie. Wien, 2010.

GMYs $2011=$ GMYs M. On the trail of a trail, the trace of a trace. Kazimierz Przerwa-Tetmajer's Cień Chopina and its compositional interpretations. Interdisciplinary Studies in Musicology 9 (2011): 215-250.

Godel 2002 = Godel B. Auf dem Weg zur Zivilgesellschaft. Frauenbewegung und Wertewandel in Russland. Frankfurt am Main, 2002. 
Goscilo 2013 = Goscilo H. Putin as Celebrity and Cultural Icon. Abingdon, 2013.

GRÜBEL 1995 = GRÜBEL R. G. Sirenen und Kometen. Axiologie und Geschichte der Motive Wasserfrau und Haarstern in slavischen und anderen europäischen Literaturen. Frankfurt am Main, 1995.

GumiLev 1991 = Гумилев Н. Собрание сочинений в 4 томах. Т. 1. Москва, 1991.

HANSEN-Löve $1989=$ HANSEN-Löve A. A. Der russische Symbolismus. System und Entfaltung der poetischen Motive. Bd. 1. Diabolischer Symbolismus. Wien, 1989.

Hermand 1989 = Hermand J. Jugendstil. Darmstadt, 1989.

HubBs 1988 = HubBs J. Mother Russia. The Feminine Myth in Russian Culture. Bloomington, 1988.

KLING 1995 = Клинг О. Стилевое становление акмеизма. Н. Гумилев и символизм. Вопросы литературы 1995/5: 101-125.

Kreß $2010=$ Kreß B. Schlüsselkonzepte der russischen Kultur und geschlechtliche Stereotypisierungen: стервизм und гламур. Zeitschrift für Slawistik 55 (2010): 127-143.

LeChner 2014 = Lechner E. Pop, Porno und Postfeminismus-Femininität und Maskulinität in den Musikvideos russischsprachiger Girlbands des 21. Jahrhunderts. Masterarbeit. Wien, 2014.

LeRMONTOV 1948 = ЛЕРмонтов М. Ю. Полное собрание сочинений. Т. 1. Москва-Ленинград, 1948.

LeŚmian 2015 = LeŚMian B. Dzieła wszystkie. Poezje zebrane. Warszawa, 2015.

ManOvich 2005 = Manovich L. Black Box-White Cube. Berlin, 2005.

MARSh 2013 = MARSH R. The Concepts of Gender, Citizenship, and Empire and Their Reflection in Post-Soviet Culture. The Russian Review 72 (2013): 187-211.

Martinez 2012 = Martinez F. The Erotic Biopower of Putinism: From Glamour to Pornography. Laboratorium 2012/3: 105-122.

Menzel 2013 = Menzel B. Russischer Glamour und die Ära Putin. Dossier Russland, 03. 06.2013. http://www.bpb.de/internationales/europa/russland/162348/analyse-russischerglamour-und-die-aera-putin? $\mathrm{p}=0$.

Mickiewicz 1953 = Mickiewicz A. Wiersze. Warszawa, 1953.

Miklosich 1864 = Miklosich F. Die Rusalien. Ein Beitrag zur slavischen Mythologie. Wien, 1864.

Monaghan 1997 = Monaghan P. Lexikon der Göttinnen. Ein Standardwerk der Mythologie. Bern, 1997.

NOHEJl 2013 = NoHEJl R. Einführung. Nation und Gender in der russischen Kultur. In: Nohejl R., Garfinkel O., Carl F., Cheauré E. (Hrsg.) Genderdiskurse und nationale Identität in Russland. Sowjetische und postsowjetische Zeit. München, 2013. 7-15.

NorwID $2000=$ NorwID C. K. Wiersze. Poezja i dobroć. Wybór z utworów. Warszawa, 2000.

Отто 2001 = Отто В. Unterwasser-Literatur. Von Wasserfrauen und Wassermännern. Würzburg, 2001.

Peverini $2010=$ Peverini P. The Aesthetics of Music Videos: An Open Debate. In: KeAzor H., WüBbena T. (Hrsg.) Rewind, Play, Fast Forward. The Past, Present and Future of the Music Video. Bielefeld, 2010. 135-153.

Puškin $1974=$ Пушкин А. С. Собрание сочинений в 10 томах. Т. 1. Москва, 1974.

PUŠKIN 1975 = Пушкин А. С. Собрание сочинений в 10 томах. Т. 4 . Москва, 1975.

RitTer 2006 = RitTer M. Präsidenten, Prinzen, Patriarchen - zur Bedeutung der Frauenbewegung für die Zivilgesellschaft in Russland. In: WECKWERT A., WischermanN U. (Hrsg.) Das Jahrhundert des Feminismus. Streifzüge durch nationale und internationale Bewegungen und Theorien. Königstein, 2006. 113-126. 
RÖSING 2003 = RösING H. Bilderwelt der Klänge - Klangwelt der Bilder. Beobachtungen zur Konvergenz der Sinne. In: Helms D., Phleps T. (Hrsg.) Clipped Differences. Geschlechterrepräsentationen im Musikvideo. Bielefeld, 2003. 9-25.

RutTEN $2010=$ RutTEN E. Unattainable Bride Russia. Gendering Nation, State, and Intelligentsia in Russian Intellectual Culture. Evanston, 2010.

RutTen 2013 = RutTen E. Putin auf Panties. Die Sexualisierung Russlands in der spätsowjetischen und postsowjetischen Kultur. In: NoHeJl R., GARFInKEL O., CARL F., ChEAuré E. (Hrsg.) Genderdiskurse und nationale Identität in Russland. Sowjetische und postsowjetische Zeit. München, 2013. 127-156.

SCHNEIDER 1970 = SCHNEIDER H. Der frühe Bal'mont. Untersuchungen zu seiner Metaphorik. München, 1970.

VÀŇA 1992 = VÀŇA Z. Mythologie und Götterwelt der slawischen Völker. Die geistigen Impulse Ost-Europas. Stuttgart, 1992.

Witkowska-PrZybylski 1997 = Witkowska A., PrZybylski R. Romantyzm. Warszawa, 1997.

Woldan 2013 = Woldan A. Das Rusalka-Motiv bei Puškin, Ševčenko und Mickiewicz ein komparatistischer Versuch. In: Wiek XIX. Rocznik Towarzystwa Literackiego im. Adama Mickiewicza. Rok VI. Warszawa, 2013. 427-453.

ZGORZELSKI 1976 = ZGORZELSKi Cz. O sztuce poetyckiej Mickiewicza. Próby zbliżeń i uogólnień. Warszawa, 1976. 